Nagoya Math. J., 221(1) (2016), 33-68

DOI $10.1017 / \mathrm{nmj} .2016 .2$

\title{
PURE SUBRINGS OF COMMUTATIVE RINGS
}

\author{
S. CHAKRABORTY, R. V. GURJAR AND M. MIYANISHI
}

Abstract. We study subalgebras $A$ of affine or local algebras $B$ such that $A \hookrightarrow B$ is a pure extension from algebraic and geometric viewpoints.

\section{$\S 0$. Introduction}

Let $\varphi: A \rightarrow B$ be a homomorphism of commutative rings. We say that $\varphi$ is a pure homomorphism, or pure embedding, or pure extension if the canonical homomorphism $\varphi_{M}: M \otimes_{A} A \rightarrow M \otimes_{A} B$ is injective, for any $A$-module $M$. This condition implies that $\varphi$ is injective. Therefore, identifying $A$ with $\varphi(A)$, we often say that $A$ is a pure subring of $B$, or that $B$ is a pure extension of $A$. The notion of purity was first raised by Warfield [15]. The associated morphism ${ }^{a} \varphi: \operatorname{Spec} B \rightarrow \operatorname{Spec} A$ is also called a pure morphism of affine schemes.

A morphism of schemes $p: Y \rightarrow X$ is called a pure morphism if $p$ is an affine morphism, and there exists an affine open covering $\left\{U_{i}\right\}_{i \in I}$ of $X$, such that $\left.p\right|_{p^{-1}\left(U_{i}\right)}: p^{-1}\left(U_{i}\right) \rightarrow U_{i}$ is a pure morphism for every $i \in I$. The formal properties of pure subalgebras and pure morphisms are summarized in Section 1.

In this paper, we are interested in pure subalgebras of an affine or local domain. The underlying field $k$, unless otherwise specified, is always assumed to be algebraically closed and of characteristic 0 . Sometimes we also assume $k$ to be the field of complex numbers $\mathbb{C}$ to apply results of local analytic algebras. Let $A$ be a pure $k$-subalgebra of an affine domain $B$. Then, by [5], $A$ itself is affine. Furthermore, if $B$ is normal then $A$ is also normal, and the associated morphism ${ }^{a} \varphi$ : Spec $B \rightarrow \operatorname{Spec} A$ is surjective by Lemma 1.1 below.

Received December 6, 2013. Revised February 13, 2014. Accepted August 15, 2014.

2010 Mathematics subject classification. Primary 14R15;Secondary 14R05.

The first author is supported by Council of Scientific and Industrial Research of India under the Shyama Prasad Mukherjee Fellowship.

(C) 2016 by The Editorial Board of the Nagoya Mathematical Journal 
It is easy to see that a faithfully flat extension of commutative rings is pure (Lemma 1.7). There are many important results about flat extensions of commutative rings by J. Frisch, A. Grothendieck, L. Gruson, M. Raynaud, and others.

The fibers of a flat morphism are all equidimensional, but the converse is not true in general. Interestingly, for a morphism between normal varieties, equidimensionality of fibers implies purity of the morphism (Lemma 2.7). Unfortunately, in general, fibers of a pure morphism do not have correct dimension. Rings of invariants of reductive group actions on a polynomial ring provide plenty of examples of pure subrings such that the corresponding quotient morphisms do not have equidimensional fibers. This fact is one of the underlying reasons why invariant theory is such a fascinating subject.

It is the authors' impression that there are not many papers about pure extensions. We use some important results due to M. Hochster about pure extensions (Section 1). In this paper we prove several new results about pure extensions, and give many examples to indicate the subtlety of the notion of purity.

We study the following questions.

Question 1. If $R \subseteq S$ is a pure extension of algebraic local rings, is the induced map of completions $\hat{R} \rightarrow \hat{S}$ also a pure homomorphism?

QUESTION 2. If $R \subseteq S$ is an extension of algebraic local rings such that the associated map Spec $S \rightarrow \operatorname{Spec} R$ is surjective, is the induced map Spec $\hat{S} \rightarrow$ Spec $\hat{R}$ also surjective?

Closely related to these is another question.

Question 3. Let $R \subseteq S$ be a pure extension of analytic local rings, corresponding to germs of complex spaces $(V, p),(W, q)$. Is it true that the image of a neighborhood of $q$ in $W$ contains a neighborhood of $p$ in $V$ ?

We show that all of these questions have affirmative answers with mild restrictions on $R$ and $S$.

We now state the main results proved in this paper.

(1) Let $R \subseteq S$ be algebraic local rings. Assume that this is a pure extension. If $R$ is either reduced, or Gorenstein, then the induced map of completions $\hat{R} \subseteq \hat{S}$ is also pure (Corollary 3.2.1). 
(2) Let $R \subseteq S$ be algebraic local rings such that $\hat{R}$ is an integral domain. If the morphism Spec $S \rightarrow \operatorname{Spec} R$ is a surjection, then Spec $\hat{S} \rightarrow \operatorname{Spec} \hat{R}$ is also a surjection (Theorem 3.4).

(3) Let $R \subseteq S$ be a pure extension of analytic local rings over $\mathbb{C}$ such that $R$ is an integral domain. Then the image of a Euclidean neighborhood of the closed point of Spec $S$ contains a Euclidean neighborhood of the closed point of $\operatorname{Spec} R$ (Theorem 3.5).

(4) Let $A \subseteq B$ be an inclusion of normal affine domains over a field $k$ such that the morphism $\operatorname{Spec} B \rightarrow \operatorname{Spec} A$ is generically finite and surjective. Then there exists a normal affine domain $C / k$ such that $A \subseteq C \subseteq B$, the morphism $\operatorname{Spec} C \rightarrow \operatorname{Spec} A$ is quasi-finite and surjective, and $C$ is the largest affine subring of $B$ containing $A$ with these properties. Further, $C$ is a pure extension of $A$ (Theorem 4.4).

The proofs of Theorems 3.4 and 3.5 are rather nontrivial. For the proof of Theorem 3.4 we need ideas from Artin's approximation theorem.

The following question arises in this context, and we discuss it later.

Question. Let $A$ be a two-dimensional pure subalgebra of $k[x, y]$. Is the associated morphism Spec $k[x, y] \rightarrow$ Spec $A$ quasi-finite?

We give a counterexample to this question. However, if $A$ is a graded subring of $k[x, y]$ then we do not yet know if the answer is affirmative.

A faithfully flat extension is pure. In general, the fibers of a pure morphism can have variable dimensions. In Section 5 we give several examples, and also some positive results, which illustrate the subtlety of the notion of pure extensions, and which shed more light on this important notion.

We raise several open problems whose solution, we believe, is valuable for algebraic geometry and commutative algebra.

We are indebted to M. Hashimoto for motivating us to study the present subject. In fact, the announcement of his result [5] at a workshop in Oberwolfach in 2005 initiated our study of pure subalgebras of polynomial rings.

\section{$\S 1 . \quad$ Preliminaries}

We recall some of the standard properties of pure extensions which are frequently used in the paper. Some of these are trivial consequences of the definitions. For the rest, appropriate references are mentioned. 
For a commutative ring $A$, let Spec $A$ and Max $A$ denote the sets of prime ideals and maximal ideals of $A$ respectively.

Let $A \subseteq B$ be commutative rings. If $A$ is a direct summand of $B$ (as an $A$-module), it follows from the definition that $A$ is a pure subring of $B$. This fact is implicitly used in this paper. Later, we will see two important instances when this happens:

(a) if $A$ is normal, and $B$ is a finite module over $A$; or

(b) if $A$ is a complete local ring, and $A \subseteq B$ is pure.

By an algebraic local ring we mean a localization of an affine algebra over a field $k$ at a maximal ideal.

Lemma 1.1. Let $\varphi: A \rightarrow B$ be a pure embedding. Then the following assertions hold.

(1) Suppose that $\varphi$ splits as

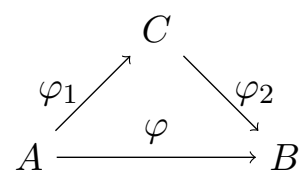

Then $\varphi_{1}$ is a pure embedding. If $\varphi_{1}$ and $\varphi_{2}$ are pure embeddings, then so is $\varphi$, that is, pure extension is transitive.

(2) For any ideal $I$ of $A$, we have $I B \cap A=I$. In particular, if $B$ is Noetherian, so is $A$.

(3) The associated morphism $\varphi^{*}: \operatorname{Spec} B \rightarrow \operatorname{Spec} A$ is surjective.

LEMMA 1.2. A ring homomorphism $\varphi: A \rightarrow B$ is pure if and only if $\varphi_{M}: M \rightarrow M \otimes B$ is injective for any finitely generated $A$-module $M$.

LEMma 1.3. (Local criteria for purity) If $\varphi: A \rightarrow B$ is a ring homomor phism, then the following are equivalent.

(1) The homomorphism $\varphi$ is pure.

(2) For any multiplicatively closed set $S \subseteq A, S^{-1} \varphi: S^{-1} A \rightarrow S^{-1} B$ is pure.

(3) For any $\mathfrak{p} \in \operatorname{Spec} A, \varphi_{\mathfrak{p}}: A_{\mathfrak{p}} \rightarrow B_{\mathfrak{p}}$ is pure.

(4) For any $\mathfrak{m} \in \operatorname{Max} A, \varphi_{\mathfrak{m}}: A_{\mathfrak{m}} \rightarrow B_{\mathfrak{m}}$ is pure.

(5) There exist $x_{1}, x_{2}, \ldots, x_{n} \in A$, generating the unit ideal, such that $\varphi_{x_{i}}$ : $A_{x_{i}} \rightarrow B_{x_{i}}$ is pure for each $i=1,2, \ldots, n$. 
LEMma 1.4. (Extension of scalars) If $A \hookrightarrow B$ is a pure extension of commutative rings, for any A-algebra $C, C \longrightarrow C \otimes_{A} B$ is pure.

Corollary 1.4.1. Given any two pure A-algebras $B$ and $C, A \hookrightarrow$ $B \otimes_{A} C$ is a pure extension.

A ring homomorphism $f: A \rightarrow B$ is called cyclically pure if, for any ideal $I \subseteq B, A \cap I B=I$, or, equivalently, if any ideal in $A$ is contracted from some ideal in $B$. Note that analogues of Lemma 1.1 hold for cyclically pure extensions.

The following important result from [6, Theorem 1.7] is often used in this paper. We state a special case which is sufficient for our purposes.

Lemma 1.5. Let $A$ be a Noetherian excellent ring, and let $\varphi: A \rightarrow B$ be an embedding. Then the following assertion holds.

If $A$ is reduced, or Gorenstein, or a local Cohen-Macaulay ring of dimension $\geqslant 2$, then $\varphi$ is a pure embedding if and only if it is cyclically pure.

The following result is proved in [9].

LEMMA 1.6. Let $A \subseteq B$ be affine domains over a field of characteristic 0 . If $A$ is normal, and the induced morphism $\operatorname{Spec} B \rightarrow \operatorname{Spec} A$ is quasi-finite and surjective, then $\varphi$ is a pure embedding.

Lemma 1.6 immediately implies that if $A$ is a normal affine subalgebra of $k\left[x_{1}, x_{2}, \ldots, x_{n}\right]$, such that the extension is integral, then this is a pure extension.

LEMMA 1.7. If $A$ is a subring of $B$, such that $B$ is a faithfully flat $A$ module, then the extension is pure.

Proof. See [11, Theorem 7.5].

The following criterion of purity can be found in [8, Corollary 5.3].

Lemma 1.8. Let $A$ be a Noetherian ring, which is a subring of $B$. Then $A$ is a pure subring of $B$ if and only if $A$ is a direct summand, as an $A$ module, of each finitely generated $A$-submodule of $B$. In particular, if $B$ is a finitely generated $A$-module, $A \hookrightarrow B$ is a pure embedding if and only if $A$ is a direct summand of $B$ as an $A$-module.

The following result and its proof were shown to us by $\mathrm{C}$. Huneke to whom we are very thankful. 
Lemma 1.9. Let $A$ be a pure subring of $B$. Assume that $A$ is a Noetherian complete local domain. Then $A$ is a direct summand of $B$ as an A-module.

Proof. Let $E$ be the injective hull of the residue field of $A$. The hypothesis that $A$ is a pure subalgebra of $B$ implies that the map $E \rightarrow E \otimes_{A} B$ is injective. Hence, the dual map $\operatorname{Hom}_{A}\left(E \otimes_{A} B, E\right) \rightarrow \operatorname{Hom}_{A}(E, E) \cong A$ is surjective, where the last isomorphism follows since $A$ is complete. However, $\operatorname{Hom}_{A}\left(E \otimes_{A} B, E\right)$ is isomorphic to $\operatorname{Hom}_{A}(B, A)$, and the surjective map from this module to $A$ just sends a homomorphism $f$ to $f(1)$. Thus, there exists an $A$-module homomorphism $g: B \rightarrow A$, and consequently $A$ is a direct summand of $B$.

\section{§2. Some new observations}

We use the following terminologies in this paper.

Let $f: X \longrightarrow Y$ be a dominant morphism of affine algebraic varieties. Then we have the following.

(1) The morphism $f$ is called pure if the induced morphism $f^{*}: \mathcal{O}_{Y} \longrightarrow$ $\mathcal{O}_{X}$ is pure.

(2) A point $x \in X$ is called pure if $\mathcal{O}_{Y, f(x)} \longrightarrow \mathcal{O}_{X, x}$ is pure. We call $f$ strongly pure if each $x \in X$ is pure.

(3) A point $y \in f(X)$ is called completely pure if each point in $f^{-1}(y)$ is pure.

(4) A point $y \in Y$ is called partially pure if some point in $f^{-1}(y)$ is pure.

(5) A point $y \in f(X)$ is called pure if $\mathcal{O}_{Y, y} \longrightarrow \mathcal{O}_{X} \otimes \mathcal{O}_{Y, y}$ is pure.

Note. An algebraic variety is always assumed to be irreducible unless otherwise specified.

Since given any $y \in f(X)$ and some $x \in f^{-1}(y)$, we have a natural factorization

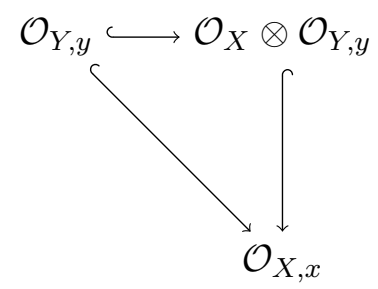

it is easy to see that any completely pure point is partially pure, which, in turn, is pure. 
Let $A \hookrightarrow B$ be a pure extension. Suppose that there is an element $x$ of $B$ satisfying a relation

$$
a_{n} x^{n}+a_{n-1} x^{n-1}+\cdots+a_{1} x+a_{0}=0, \quad \text { with } a_{i} \in A, \quad \forall i .
$$

Then, as an easy corollary of Lemma 1.5, it follows that $a_{0}$ is contained in the ideal generated by $a_{1}, a_{2}, \ldots, a_{n}$ in $A$. A partial converse of this fact holds under special conditions.

Lemma 2.1. Let $A$ be a Dedekind domain. Then, for any polynomial

$$
f(x):=a_{0}+a_{1} x+a_{2} x^{2}+\cdots+a_{n} x^{n} \in A[x] \text { of positive degree, }
$$

$A \longrightarrow A[x] /(f)$ is pure if $a_{0}$ is contained in the ideal generated by $a_{1}, a_{2}, \ldots, a_{n}$ in $A$.

Proof. In view of the local criteria for purity as in Lemma 1.3, after localizing the extension at a maximal ideal of $A$, we may assume that $A$ is a discrete valuation ring. First, we observe that, if $\mathrm{B}$ is a (cyclically) pure A-algebra, for any A-algebra C, $B \oplus C$ is also a (cyclically) pure A-algebra.

Now, coming to the proof, if $a_{0}=0$, there exists $m>0$ such that $x^{m} \mid f$ but $x^{m+1}$ does not divide $f$. Since $A[x]$ is a unique factorization domain, and $\operatorname{gcd}\left(x^{m}, f / x^{m}\right)=1$, we have

$$
A \hookrightarrow \frac{A[x]}{(f)} \hookrightarrow \frac{A[x]}{\left(x^{m}\right)} \oplus \frac{A[x]}{\left(f / x^{m}\right)} .
$$

Now, $A[x] /\left(x^{m}\right)$, being a free module over $\mathrm{A}$, is pure. Hence, by the above observation,

$$
A \hookrightarrow \frac{A[x]}{\left(x^{m}\right)} \oplus \frac{A[x]}{\left(f / x^{m}\right)}
$$

is also pure, and consequently so is $A \hookrightarrow A[x] /(f)$.

Otherwise, if $a_{0} \neq 0$, then $f$ can be factored as $f=a g$, where $a:=$ $\operatorname{gcd}\left(a_{i}\right)_{i=0}^{n}$, and $g$ is a primitive polynomial. Therefore, we have

$$
\frac{A[x]}{(f)}=\frac{A[x]}{(a g)} \hookrightarrow \frac{A}{(a)}[x] \oplus \frac{A[x]}{(g)} .
$$

However, then, by [14], $A[x] /(g)$ is a faithfully flat A-algebra. Therefore, the above observation, together with Lemma 1.7, implies that

$$
A \hookrightarrow \frac{A}{(a)}[x] \oplus \frac{A[x]}{(g)}
$$

is pure, and consequently so is $A \hookrightarrow A[x] /(f)$. 
The following lemma is in the same spirit, which shows that for Dedekind domains, the question of purity boils down to the question of surjectivity of the associated morphism of schemes.

Lemma 2.2. Let $A \subseteq B$ be integral domains. Suppose that a height 1 prime $\mathfrak{p}$ in $A$ is contracted from $B$, and that $A_{\mathfrak{p}}$ is a DVR. Then any $\mathfrak{p}$ primary ideal in $A$ is contracted from some ideal in $B$.

Proof. We have the following commutative diagram:

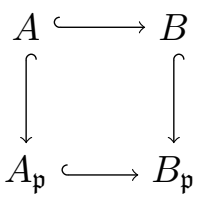

Since $\mathfrak{p}$-primary ideals in $A$ are in one-to-one correspondence with the $\mathfrak{p} A_{\mathfrak{p}}$-primary ideals of $A_{\mathfrak{p}}$, it is sufficient to prove that any $\mathfrak{p} A_{\mathfrak{p}}$-primary ideal is contracted from some ideal in $B_{\mathfrak{p}}$. This is a special case of the following lemma.

Lemma 2.3. Let $A \subseteq B$ be integral domains, with $A$ being a UFD. If any height 1 prime in $A$ is contracted from some ideal in $B$, then any principal ideal in $A$ is a contracted ideal.

Proof. Let $a$ be any nonzero, nonunit element in $A$ with a prime factorization (up to a unit)

$$
a=\prod_{i=1}^{r} p_{i}^{e_{i}}, \quad \text { with distinct primes } p_{i} \text { and positive integers } e_{i} .
$$

If possible, let $a^{*} \in(a) B \cap A-(a)$. Then $a^{*}$ can be written as

$$
a^{*}=\beta \prod_{i=1}^{r} p_{i}^{f_{i}}
$$

where the $f_{i}$ are nonnegative integers, and $\beta \in A-\{0\}$ is relatively prime to $p_{i}$, for each $i$. Since $a^{*} \notin(a)$, there exists some $i$ for which $f_{i}<e_{i}$. Again, $a^{*} \in(a) B$ implies that there exists some $b \in B$ such that $a^{*}=a b$, implying that $\beta \prod_{j \neq i} p_{j}^{f_{j}} \in\left(p_{i}\right)$, leading to a contradiction.

We have the following corollaries of Lemma 2.2 (using Lemma 1.5). 
Corollary 2.3.1. If $A \subseteq B$ are integral domains, with $A$ being a Noetherian normal domain, such that every height 1 prime in $A$ is contracted, then any unmixed ideal of height 1 in $A$ is also a contracted ideal.

COROLlary 2.3.2. Any surjective morphism from an affine variety to a smooth curve is pure.

This also follows easily since such a morphism is faithfully flat (and Lemma 1.7).

The following corollary extends a cyclically pure version of Lemma 2.1 to the formal power series case.

Corollary 2.3.3. Let $A$ be a Dedekind domain, and let $f \in A[[x]]$ be a nonconstant formal power series. Then

$$
A \hookrightarrow \frac{A[[x]]}{(f)}
$$

is cyclically pure if $f_{0}$ lies in the ideal generated by $f_{1}, f_{2}, \ldots$, where $f(x):=$ $f_{0}+f_{1} x+f_{2} x^{2}+\cdots$.

Proof. The proof follows almost the same lines as the proof of Lemma 2.1. However, instead of using Nagata's criteria for faithful flatness, we take a prime factorization of $f$ and apply Corollary 2.3.1.

The following lemma is a special case of Lemma 1.1(1). We write it down in this particular form for the sake of future reference.

Lemma 2.4. (Square diagram for purity) If

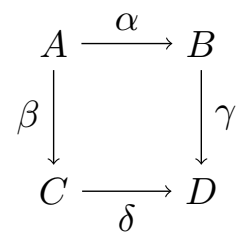

is a commutative diagram of commutative rings, then $\beta, \gamma, \delta$ are (cyclically) pure implies that $\alpha$ is also (cyclically) pure.

Proof. The proof follows directly from the definitions.

Lemma 2.5. Let $A \subseteq B$ be integral domains, with $A$ being a Noetherian normal domain. If the image of Spec $B$ contains all height 1 primes of Spec $A$, then any element $\xi$ of the quotient field of $A$, which is integral over 
$B$, is an element of $A$. In particular, the result is true for pure extensions, as the morphism of schemes associated to any pure morphism is surjective.

Proof. Since $A$ is normal, it suffices to show that $\xi$ is in every localization of $A$ at a height 1 prime ideal $\mathfrak{p}$. Suppose that this is not true for some $\mathfrak{p}$. Then $\xi^{-1}$ must lie in the maximal ideal $\mathfrak{p} A_{\mathfrak{p}}$, that is, $\xi=1 / a$ for some $a \in \mathfrak{p} A_{\mathfrak{p}}$. Since $\xi$ is integral over $B, \xi$ satisfies an integral equation $\xi^{n}+$ $b_{1} \xi^{n-1}+\cdots+b_{n}=0$, with $b_{i} \in B$. By replacing $\xi$ by $1 / a$, and clearing the denominator in the equation, we obtain

$$
1+b_{1} a+b_{2} a^{2}+\cdots+b_{n} a^{n}=0 .
$$

Therefore, $a$ is a unit in $B$. However, by the given condition, there exists $\mathfrak{q} \in$ Spec $B$ such that $\mathfrak{p}=\mathfrak{q} \cap A$, which implies that $a \in \mathfrak{q}$, leading to a contradiction.

Lemma 2.6. Let $A$ be an affine $k$-domain, and let $A \hookrightarrow B$ be a pure embedding. Let $I$ be a radical ideal of $A$, let $\bar{A}=A / I$, and let $\bar{B}=B / I B$. Then $\bar{A} \hookrightarrow \bar{B}$ is a pure embedding.

More generally, if $I$ is any ideal in $A$ such that $\bar{A}$ is Gorenstein, then $\bar{A} \hookrightarrow \bar{B}$ is a pure embedding.

Proof. It is a special case of the base change property of pure extensions, as stated in Lemma 1.4.

Lemma 2.7. Let $\varphi: X \longrightarrow Y$ be a dominant morphism of affine varieties, with $\operatorname{dim} X=m$ and $\operatorname{dim} Y=n$. Assume that $Y$ is normal. Let $x \in X$ be such that any irreducible component of $\varphi^{-1}(\varphi(x))$, which contains $x$, has dimension equal to $m-n$. Then the induced local morphism

$$
\varphi_{x}^{*}: \mathcal{O}_{Y, \varphi(x)} \longrightarrow \mathcal{O}_{X, x}
$$

is pure.

Proof. We indicate a proof when $m-n=1$. The general case is proved similarly by taking repeated hyperplane sections. Let $H$ be a general hyperplane section of $X$ through $x$. Then $\left.\varphi\right|_{H}: H \longrightarrow Y$ is quasi-finite. Hence, the induced morphism of complete local rings $\hat{\mathcal{O}}_{Y, \varphi(x)} \longrightarrow \hat{\mathcal{O}}_{H, x}$ is finite. By Lemma 1.8, this is a pure embedding; in fact this is a direct summand. Since there is a surjection $\hat{\mathcal{O}}_{X, x} \longrightarrow \hat{\mathcal{O}}_{H, x}$, we deduce that $\hat{\mathcal{O}}_{Y, \varphi(x)} \longrightarrow \hat{\mathcal{O}}_{X, x}$ is also a direct summand, hence a pure extension. This, together with Lemmas 2.4 and 1.7, implies that $\mathcal{O}_{Y, \varphi(x)} \longrightarrow \mathcal{O}_{X, x}$ is a pure extension. 
REMARK. In Section 5.10 we give an example where the set of completely pure points is not open in $Y$.

We add here the following remark.

Let $G$ be a reductive algebraic group acting on an affine regular $\mathbb{C}$ domain $A$. Then by Hochster-Roberts [7], the ring $A^{G}$ of $G$-invariants is a pure subalgebra of $A$. In a similar context, we can ask whether the ring of invariants of a $G_{a}$-action on a polynomial ring $\mathbb{C}\left[x_{1}, \ldots, x_{n}\right]$ is a pure subring of $\mathbb{C}\left[x_{1}, \ldots, x_{n}\right]$. The answer is positive if $n \leqslant 3$ but negative for $n \geqslant 4$. There are three crucial points to prove in the case $n \leqslant 3$. Namely, we have the following.

(1) The ring of invariants is regular.

(2) The quotient morphism

$$
q: \operatorname{Spec} \mathbb{C}\left[x_{1}, \ldots, x_{n}\right] \rightarrow \operatorname{Spec} \mathbb{C}\left[x_{1}, \ldots, x_{n}\right]^{G_{a}}
$$

is surjective.

(3) The fibers of the quotient morphism $q$ are all of dimension one.

In fact, with all three conditions satisfied, $q$ is a faithfully flat morphism, and hence pure by Lemma 1.7. In the study of pure extensions, the surjectivity of the morphism $\operatorname{Spec} B \rightarrow \operatorname{Spec} A$ is always a key point. Bonnet [3] and later Kaliman [10] proved via topological arguments that if $n=3$, the quotient morphism $q$ is surjective. This is a fairly significant result in understanding the subalgebras of a polynomial ring, and it is desirable to give a proof which may elucidate the algebro-geometric background.

Proposition 2.8. Let $B=\mathbb{C}\left[x_{1}, \ldots, x_{n}\right]$ be a polynomial ring with a nontrivial action of the additive group $G_{a}$, let $D$ be the associated locally nilpotent derivation, and let $A$ be the kernel of $D$. Then we have the following assertions.

(1) If $n \leqslant 3$, the inclusion $A \hookrightarrow B$ is a pure embedding.

(2) Suppose $n=4$. Define the Weitzenböck derivation by $D\left(x_{i}\right)=x_{i+1}$ for $1 \leqslant i \leqslant 3$ and $D\left(x_{4}\right)=0$. Then the quotient morphism $q: \operatorname{Spec} B \rightarrow$ Spec $A$ is neither surjective nor flat. Hence, the inclusion $A \hookrightarrow B$ is not a pure embedding.

Proof. (1) If $n \leqslant 3$, we show that $B$ is faithfully flat over $A$, whence $A \hookrightarrow B$ is a pure embedding by Lemma 1.7 . We only consider the case 
$n=3$. It is known that $A$ is a polynomial ring in two variables, and $q$ is equidimensional of dimension one (cf. [12]). Hence, $B$ is flat over $A$. On the other hand, the surjectivity of $q$ is proved by [3] and [10]. Hence, $B$ is faithfully flat over $A$.

(2) The ring $A$ is generated over $\mathbb{C}$ by the following four elements:

$$
\begin{gathered}
X=x_{4}, Y=x_{2} x_{4}-\frac{1}{2} x_{3}^{2}, Z=x_{1} x_{4}^{2}-x_{2} x_{3} x_{4}+\frac{1}{3} x_{3}^{3}, \\
W=9 x_{1}^{2} x_{4}^{2}+\left(8 x_{2}^{2}-18 x_{1} x_{3}\right) x_{2} x_{4}+6 x_{1} x_{3}^{3}-3 x_{2}^{2} x_{3}^{2} .
\end{gathered}
$$

Hence, Spec $A$ is a hypersurface $X^{2} W=(2 Y)^{3}+(3 Z)^{2}$ in $\mathbb{A}^{4}$. We show that the set $\{(0,0,0, W) \mid W \neq 0\}$ is not in the image of $q$. In fact, for a closed point of Spec $A$ with $X=x_{4}=0$, we have $Y=-\frac{1}{2} x_{3}^{2}$ and $Z=\frac{1}{3} x_{3}^{3}$. If $x_{3}$ moves, the point $\left(-\frac{1}{2} x_{3}^{2}, \frac{1}{3} x_{3}^{3}\right)$ covers the curve $(2 Y)^{3}+(3 Z)^{2}=0$ in the $(Y, Z)$-plane. If $x_{3} \neq 0$, we can find $x_{1}, x_{2}$ so that $W=6 x_{1} x_{3}^{3}-3 x_{2}^{2} x_{3}^{2}$ takes an arbitrary value. If $x_{3}=0$, then $W=0$. Hence, $\{(0,0,0, W) \mid W \neq 0\}$ is not in the image of $q$.

As for the flatness of $B$ over $A$, it suffices to show that $q$ is not equidimensional. It is clear that the general fibers of $q$ have dimension one. However, over the point $(0,0,0,0)$, the fiber is given by $\left\{x_{3}=x_{4}=0\right\}$, which is a plane. Therefore, we have shown that $A \hookrightarrow B$ is not a pure embedding.

A more straightforward way to see that $A \hookrightarrow B$ is not a pure embedding is to use that $I B \cap A \neq I$, for $I:=X A+Y A$. In fact, $I B$ contains $X, Y, Z, W$, but $Z, W \notin I$.

We note that Bonnet [3] constructed an example of $G_{a}$-action on $\mathbb{A}^{4}$ whose quotient morphism is not surjective.

\section{$\S 3 . \quad$ Purity and surjectivity}

In this section we study the relationship between the notions of purity and surjectivity of the induced morphism Spec $S \rightarrow$ Spec $R$ for an inclusion of rings $R \hookrightarrow S$. Of particular interest will be the cases when $R, S$ are analytic local rings, or complete local rings.

We now answer the questions raised in the introduction.

Let $R \subseteq S$ be algebraic local rings.

By Lemma 1.7, faithful flatness implies purity. Therefore, in answering Question 3, we have a generalization of the well-known result that flat maps are open.

We believe that this is a new result about pure morphisms. The proof of this result is quite nontrivial. 
As a first step in relating purity to surjectivity we record the following easy consequence of Chevalley's theorem, as in [11, Theorem 6], which tells us that the surjectivity of the Spec map, associated to a local morphism, actually translates into the local surjectivity in Zariski-topology.

THEOREm 3.1. Let $A \subseteq B$ be Noetherian rings of finite krull dimension, such that $A$ is an integral domain, and $B$ is a finitely generated algebra over $A$. Suppose that, for some $\mathfrak{p} \in \mathrm{Spec} B$, the induced morphism of schemes

$$
\text { Spec } B_{\mathfrak{p}} \longrightarrow \text { Spec } A_{\mathfrak{p}^{\mathfrak{c}}}
$$

is surjective. Then there exists an open set $V \subseteq$ Spec $A$, such that $\mathfrak{p}^{\mathfrak{c}} \in V \subseteq$ ${ }^{a} i(\operatorname{Spec} B)$, where ${ }^{a} i: \operatorname{Spec} B \rightarrow$ Spec $A$ is induced by the natural inclusion $i: A \longrightarrow B$.

COROLlary 3.1.1. Let $\phi: X \longrightarrow Y$ be a morphism of affine algebraic sets, with $Y$ being irreducible. If $x \in X$ is such that

$$
\operatorname{Spec} \mathcal{O}_{X, x} \longrightarrow \operatorname{Spec} \mathcal{O}_{Y, \phi(x)}
$$

is surjective, then there exists an open set $V \subseteq Y$, satisfying $\phi(x) \in V \subseteq$ $\phi(X)$. In particular, the result is true when $x \in X$ is pure.

The next result shows that purity carries over to completion.

THEOREM 3.2. Let $R_{\mathfrak{m}} \longrightarrow S_{\mathfrak{n}}$ be a cyclically pure extension of algebraic local rings. Then the induced map $\hat{R}_{\mathfrak{m}} \rightarrow \hat{S}_{\mathfrak{n}}$ is also cyclically pure. (Note that cyclic purity implies that the morphism is local.)

Proof. If $\psi: \hat{R}_{\mathfrak{m}} \rightarrow \hat{S}_{\mathfrak{n}}$ is the induced natural map, then we get the following commutative diagram:

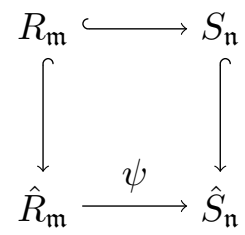

Given any ideal $\hat{I}$ of $\hat{R}_{\mathfrak{m}}$, by Krull's intersection theorem, we have

$$
\hat{I}=\bigcap_{n=1}^{\infty}\left(\hat{I}+\hat{\mathfrak{m}}^{n}\right) .
$$


Since the ideal $\hat{I}+\hat{\mathfrak{m}}^{n}$ is $\hat{\mathfrak{m}}$-primary for each $n$, it is sufficient to show that any $\hat{\mathfrak{m}}$-primary ideal is contracted from some ideal in $\hat{S}_{\mathfrak{n}}$. If $\hat{\mathfrak{q}}$ is any $\hat{\mathfrak{m}}$ primary ideal in $\hat{R}_{\mathfrak{m}}$, then there exists an $\mathfrak{m}$-primary ideal $\mathfrak{q}$ in $R_{\mathfrak{m}}$ such that $\hat{\mathfrak{q}}=\mathfrak{q} \hat{R}_{\mathfrak{m}}$. Now, $R_{\mathfrak{m}} \hookrightarrow S_{\mathfrak{n}}$ being pure, there exists an ideal $\mathfrak{p} \in S_{\mathfrak{n}}$ satisfying $\mathfrak{p} \cap R_{\mathfrak{m}}=\mathfrak{q}$. Since completion is faithfully flat and hence pure by Lemma 1.7, there exists an ideal $\hat{\mathfrak{p}} \in \hat{S_{\mathfrak{n}}}$ such that $\hat{\mathfrak{p}} \cap S_{\mathfrak{n}}=\mathfrak{p}$. The diagram being commutative, $\psi^{-1}(\hat{\mathfrak{p}}) \cap R_{\mathfrak{m}}=\mathfrak{q}$. Therefore, $\psi^{-1}(\hat{\mathfrak{p}})$ is also $\hat{\mathfrak{m}}$-primary, and consequently an extended ideal. Hence, $\psi^{-1}(\hat{\mathfrak{p}})=\hat{\mathfrak{q}}$, as the extended ideals are in one-to-one correspondence with the contracted ideals. Therefore, $\psi: \hat{R}_{\mathfrak{m}} \rightarrow \hat{S}_{\mathfrak{n}}$ is cyclically pure.

As a corollary, we get an affirmative answer to Question 1. This also follows from [7, Proposition 6.11], but we have given a direct elementary proof.

Corollary 3.2.1. Let $R \subseteq S$ be a pure extension of algebraic local rings. If $R$ is either reduced or Gorenstein, then the induced map $\hat{R} \rightarrow \hat{S}$ is also a pure extension. Similarly, the extension of the associated analytic local rings $R_{a n} \rightarrow S_{a n}$ is also a pure extension.

Proof. The proof follows from Lemma 1.5.

Since $\hat{R}$ is the completion of $R_{a n}$, we deduce the result that the extension of the associated analytic local rings $R_{a n} \rightarrow S_{a n}$ is also a pure extension.

Next, for Question 2, we begin by recalling a simple fact in commutative algebra.

LEMMA 3.3. If $\mathfrak{p}$ is a prime ideal in a Noetherian ring $R$, such that Spec $(R / \mathfrak{p})$ is infinite, then

$$
\mathfrak{p}=\bigcap_{\mathfrak{q} \in V(\mathfrak{p}) \backslash\{\mathfrak{p}\}} \mathfrak{q}
$$

Proof. The proof follows from the prime avoidance theorem.

Now, let $R$ be an algebraic local domain of dimension $>1$. Then, by a standard result in commutative algebra, $\hat{R}$ is a reduced ring. Therefore, in this case Lemma 3.3 has the following corollaries.

COROLLARY 3.3.1. With the above hypothesis for $R$, the intersection of all nonzero prime ideals is (0) in $R$ as well as in $\hat{R}$.

COROLlary 3.3.2. If $\operatorname{dim} \hat{R}=d$, then any prime ideal $\mathfrak{p}$ of height smaller than $d$ is the intersection of all prime ideals of height $d-1$ 
containing $\mathfrak{p}$. (Geometrically, this means that every irreducible germ of an algebroid variety is the union of all the algebroid curves in it.)

TheOREm 3.4. Let $(R, \mathfrak{m}) \subset(S, \mathfrak{n})$ be algebraic local domains such that $\hat{R}$ is an integral domain. Suppose that the morphism $\operatorname{Spec} S \rightarrow \operatorname{Spec} R$ is surjective. Then the morphism $\operatorname{Spec} \hat{S} \rightarrow \operatorname{Spec} \hat{R}$ is also surjective.

Proof. By [1, Chapter 3, (10.14)], we have an injection $\hat{R} \rightarrow \hat{S}$.

Consider first the case when $\mathfrak{m} S$ is primary for $\mathfrak{n}$. Then it is easy to see that $\hat{S}$ is integral over $\hat{R}$. In this case the result is well known.

So we assume that $\mathfrak{m} S$ is not primary for $\mathfrak{n}$. Let $d:=\operatorname{dim} R$. We further assume that $d>1$, since the result is trivial for $d=1$.

We claim that it suffices to prove that given any prime ideal $\hat{\mathfrak{p}} \subset \hat{R}$ such that height $\hat{\mathfrak{p}}=d-1$, there is a prime ideal $\hat{\mathfrak{q}} \subset \hat{S}$ such that $\hat{\mathfrak{p}}=\hat{\mathfrak{q}} \cap \hat{R}$.

For, assuming this, if $\hat{\mathfrak{p}} \in \operatorname{Spec} \hat{R}$ is of height less than $d-1$, then, by Corollary 3.3.2, there exists an ideal $I$ in $\hat{S}$ contracting to $\hat{\mathfrak{p}}$. However, any maximal element of the set $\{I \triangleleft \hat{S} \mid I \cap \hat{R}=\hat{\mathfrak{p}}\}$ is a prime ideal in $S$, and hence $\hat{\mathfrak{p}}$ must be in the image of Spec $\hat{S}$.

Let $\mathfrak{m} S=Q_{1} \cap Q_{2} \cap \cdots \cap Q_{r+1}$, where $Q_{i}$ are primary for prime ideals $\mathfrak{p}_{\mathfrak{i}}$ strictly smaller than $\mathfrak{n}$ for $1 \leqslant i \leqslant r$, and $Q_{r+1}$ (if it occurs in a minimal primary decomposition) is primary for $\mathfrak{n}$. Suppose that $\hat{\mathfrak{p}}$ is not of the form $\hat{\mathfrak{q}} \cap \hat{R}$ for any prime ideal of $\hat{\mathfrak{q}}$ in $S$. Then $\hat{\mathfrak{p}} \hat{S}=Q_{1}^{\prime} \cap \cdots \cap Q_{s}^{\prime} \cap Q_{s+1}^{\prime}$, where $Q_{j}^{\prime}$ is primary for a prime ideal $\mathfrak{p}_{j}^{\prime}$ which contracts to some $\mathfrak{p}_{j_{i}}$ for $1 \leqslant j \leqslant$ $s, j_{i} \leqslant r$, and $Q_{s+1}^{\prime}$ is primary for $\hat{\mathfrak{n}}$. Clearly, $s \geqslant 1$.

We write $S=k\left[x_{1}, \ldots, x_{n}\right]_{\left(x_{1}, \ldots, x_{n}\right)}, R=k\left[f_{1}, \ldots, f_{e}\right]_{\left(f_{1}, \ldots, f_{e}\right)}$. It follows that $f_{l} \in \mathfrak{p}_{i}$ for each $l$, and $1 \leqslant i \leqslant r+1$, where $\mathfrak{p}_{r+1}=\mathfrak{n}$. Let $\hat{\mathfrak{p}}=$ $\left(\varphi_{1}, \ldots, \varphi_{m}\right) \subset k\left[\left[f_{1}, \ldots, f_{e}\right]\right]$. Then $\varphi_{j} \in \mathfrak{p}_{\beta}^{\prime}$ for each $j$, and $\beta \leqslant s+1$. Each $\mathfrak{p}_{\beta}^{\prime}$ contains some $\mathfrak{p}_{\beta_{i}}$. Hence, each $f_{\alpha}$ is contained in each $\mathfrak{p}_{\beta}^{\prime}$ for $\beta \leqslant s+1$. Thus, $\varphi_{j} \in Q_{\beta}^{\prime}$ for each $j$, and $\beta \leqslant s+1$.

There exists $N>0$ such that $\mathfrak{p}_{\beta}^{\prime}{ }^{N} \subset Q_{\beta}^{\prime}$ for each $\beta$. Hence, we can write

$$
\varphi_{i}=\varphi_{j 0}+\varphi_{j 1}
$$

where each $\varphi_{j 0}$ is a polynomial in $f_{1}, f_{2}, \ldots, f_{e}$, and $\varphi_{j 1} \in \hat{\mathfrak{n}} \cdot Q_{\beta}^{\prime}$. It follows that the ideal $\left(\varphi_{j o}, \ldots, \varphi_{m 0}\right)$ generates $\cap_{1}^{s+1} Q_{\beta}^{\prime} \bmod \hat{\mathfrak{n}} \cdot\left(\cap_{1}^{s+1} Q_{\beta}^{\prime}\right)$. By Nakayama's lemma, this implies that $\left(\varphi_{10}, \ldots, \varphi_{m 0}\right)=\hat{\mathfrak{p}} \hat{S}$.

By $[2$, pages $32-33]$, we can assume that $\left(\varphi_{10}, \ldots, \varphi_{m 0}\right) \hat{R}$ is of height $d-1$. Let $I=\left(\varphi_{10}, \ldots, \varphi_{m 0}\right) \subset R$. 


\section{Claim. $I S \cap R \subset \sqrt{I}$.}

To see this, we write the primary decomposition of $I$ as $I=\cap J_{i}$, where the $J_{i}$ are primary for distinct prime ideals $\widetilde{J}_{i}$. Since Spec $S \rightarrow \operatorname{Spec} R$ is surjective, we have $\widetilde{J}_{i} S \cap R=\widetilde{J}_{i}$. It follows that $I S \cap R \subset \cap \widetilde{J}_{i}=\sqrt{I}$.

Now, $(I S) \hat{S} \cap S=I S$, since $\hat{S}$ is a faithfully flat extension of $S$. It follows that $I \hat{S} \cap \hat{R}=(\hat{\mathfrak{p}} \hat{S}) \cap \hat{R}$ is an ideal, which contracts to an ideal contained in $\sqrt{I}$. Hence, $\hat{\mathfrak{p}} \hat{S}$ cannot contract to an ideal which is primary for $\hat{\mathfrak{n}}$.

This contradiction shows that $\hat{\mathfrak{p}}$ is contracted from $\hat{S}$. This completes the proof of Theorem 3.4.

REMARK. With the notation and assumptions of Theorem 3.4, we deduce easily that the morphism Spec $S_{a n} \rightarrow \operatorname{Spec} R_{a n}$ is also surjective.

The next result gives an affirmative answer to Question 3 for morphisms of complex analytic varieties. This generalizes the well-known result that a flat morphism is open.

We need the following well-known result from complex analysis.

RouchÉ's THEOREM. Let $g(w), h(w)$ be holomorphic functions of one variable $w$ in a simply connected open set $U \subseteq \mathbb{C}$. Let $\Gamma$ be a simple closed curve in $U$. If $|g|>|h|$ on $\Gamma$, then $g$ and $g+h$ have the same number of zeros counted with multiplicity inside $\Gamma$.

We use this as follows.

Let $g(z, w)$ be holomorphic in variables $z_{1}, z_{2}, \ldots, z_{r}$ and one variable $w$ in an open neighborhood $U$ of $(0,0)$ in $\mathbb{C}^{r+1}$. Suppose that $g=b_{0}(z)+$ $b_{1}(z) w+\cdots+b_{n}(z) w^{n}+\cdots$, where $n$ is the smallest positive integer such that $b_{n}$ is a unit in the convergent power series ring $\mathbb{C}\{z\}$. Assume for simplicity that $b_{n}=1+c_{n}$, where $c_{n}(z)$ is a nonunit.

Claim. There exist open sets $U_{1}, U_{2}$ in $\mathbb{C}^{r}, \mathbb{C}$ respectively such that $U_{1} \times U_{2}$ is contained in $U$, and such that for any fixed $(z) \in U_{1}$, we have $\left|b_{0}+b_{1} w+\cdots+w^{n}\right|>\left|c_{n} w^{n}+b_{n+1} w^{n+1}+\cdots\right|$ on the boundary of $U_{2}$.

To see this, suppose $|w|=\delta<1 / 2$. Since $c_{n}+b_{n+1} w+b_{n+2} w^{2}+\cdots$ vanishes at the origin in $\mathbb{C}^{r+1}$, by continuity we can assume that $\delta_{1}, \delta$ are so small that $\left|z_{i}\right|<\delta_{1}$ in $N_{1},|w|<\delta$ in $U_{2}$, and $\left|c_{n}+b_{n+1} w+\cdots\right|<1 / 2$ in $N_{1} \times U_{2}$. Since $b_{0}, b_{1}, \ldots, b_{n-1}$ are nonunits, we can find an open neighborhood $N_{1}^{\prime}$ of $0 \in C^{r}$ such that $\left|\left(b_{0}+b_{1} w+\cdots+b_{n-1} w^{n-1}\right) / w^{n}\right|<1 / 2$ for all $(z) \in N_{1}^{\prime}$ and $|w|=\delta$. Taking $U_{1}=N_{1} \cap N_{1}^{\prime}$, we get the required result. 
Now by Rouché's theorem, for any fixed $(z) \in U_{1}$, the numbers of zeroes of $b_{0}+b_{1} w+\cdots+w^{n}$ and $g$ in $U_{2}$ are the same. Clearly, by shrinking $U_{1}$ if necessary, for any $(z) \in U_{1}$ the equation $b_{0}+b_{1} w+\cdots+w^{n}=0$ has $n$ zeroes in $U_{2}$. Hence, this is also true for $g$ inside $U_{2}$. This proves that the image of any neighborhood of $(0,0)$ in $\{g=0\}$ under the map $(z, w) \rightarrow(z)$ contains a neighborhood of 0 in $\mathbb{C}^{r}$.

In the application of the above ideas in the proof of Theorem 3.5 when $\operatorname{dim} V>1$, we have $b_{n}=c_{0}+c_{n}$, where $c_{0} \in \mathbb{C}$, with $1 / 2 \leqslant\left|c_{0}\right| \leqslant 1$, and $c_{n}$ is a nonunit. Then the rest of the above argument can be changed to get an upper bound on $|w|$ which is a root of $g=0$.

TheOREM 3.5. Let $\pi:(W, q) \rightarrow(V, p)$ be a morphism of complex analytic germs. Assume that $V$ is reduced and irreducible (but $W$ may be reducible or nonreduced). If the induced map on the local rings $R:=$ $\mathcal{O}_{V, p} \rightarrow S:=\mathcal{O}_{W, q}$ is pure, then for any Euclidean neighborhood $U$ of $q$ in $W$ the image $\pi(U)$ contains a Euclidean neighborhood of $p$ in $V$.

Proof. We prove the result using suitable induction on $\operatorname{dim} V$ and the number $r+m$ defined below for $W$.

We implicitly use the following observation. If $I \subseteq R$ is an ideal such that one of the conditions of Lemma 1.5 is satisfied for the inclusion $R / I \subseteq S / I S$, then this is also a pure embedding.

We assume that $p, q$ are the origins in $\mathbb{C}^{r}, \mathbb{C}^{r+m}$ respectively.

Let $R=\mathbb{C}\left\{z_{1}, z_{2}, \ldots, z_{r}\right\} / I, S=\mathbb{C}\left\{z_{1}, \ldots, z_{r}, w_{1}, \ldots, w_{m}\right\} / J$. Here, $r+m$ may be larger than the embedding dimension of $S$. Let $J=$ $\left(f_{1}, \ldots, f_{l}\right)$.

Write $f_{i}=a_{i 0}+a_{i 1}(z) w^{A_{1}}+a_{i 2}(z) w^{A_{2}}+\cdots$, where each $A_{j}$ is a multiindex $\left(j_{1}, \ldots, j_{m}\right)$, and $w^{A_{j}}=w_{1}^{j_{1}} \cdots w_{m}^{j_{m}}$, etc. For any multi-index $A_{j}$, we denote $\left|A_{j}\right|=\sum_{a} j_{a}$.

Let $U$ be the set of points in $\mathbb{C}^{r+m}$ such that $\left|z_{i}\right| \leqslant \delta_{0},|w| \leqslant \delta_{0}<1 / 2$ for a suitable $\delta_{0}$.

Case 1. Suppose that, for some $f_{i}$, one of the coefficients $a_{i \alpha}$ is a unit in $R$. (This includes the case when $W$ is a product of $V$ and another variety.)

By making a unitary (linear) change of coordinates involving only $w_{1}, \ldots, w_{m}$ (so that Euclidean distances are preserved), we can assume that the monomial corresponding to $a_{i \alpha}$ is $w_{m}^{j_{m}}$ for some $j_{m}$. By the Weierstrass preparation theorem, we can write $f_{i}=u_{i} g_{i}$, where $u_{i}$ is a 
unit in $\mathbb{C}\left\{z_{1}, \ldots, w_{m}\right\}$, and $g_{i}=w_{m}^{j_{m}}+b_{m-1} w_{m}^{j_{m}-1}+\cdots+b_{0}$. The coefficients $b_{0}, b_{1}, \ldots, b_{m-1}$ are nonunits in $\left\{z_{1}, \ldots, z_{r}\right\}$. It follows that $S$ is integral over a subring $S_{1}$ such that $R \subseteq S_{1} \subseteq S$ and $S_{1}:=$ $\mathbb{C}\left\{z_{1}, \ldots, w_{1}, \ldots, w_{m-1}\right\} / J_{1}$. Let $W_{1}$ be the germ in $\mathbb{C}^{r+m-1}$ corresponding to $J_{1}$. Then the image of any neighborhood of $(0,0) \in W$ under the map $(z, w) \rightarrow\left(z, w_{1}, \ldots, w_{m-1}\right)$ contains a neighborhood of the origin in $W_{1}$. Any such point in $W$ is contained in $\left\{f_{i}=0\right\}$.

Now we are in a position to use Rouché's theorem. By the discussion above, we can find open neighborhoods $U_{1}, U_{2}$ of the origins in $\mathbb{C}^{r+m-1}, \mathbb{C}$ respectively such that over any point in $W_{1} \cap U_{1}$ there are exactly $j_{m}$ points in $\left(U_{1} \times U_{2}\right) \cap\left\{f_{i}=0\right\}$, one of which lies in $W$.

Since $R \rightarrow S$ is pure, so is $R \rightarrow S_{1}$. By induction, the result is true for $W_{1} \rightarrow V$. Hence, it is also true for $W \rightarrow V$.

To complete the induction for Case 1 , we must prove the result for $m=1$.

However, the same argument as above shows that in this case the morphism $\pi$ satisfies the assertion of the theorem.

Case 2. Now we can assume that for each $i$ every $a_{i \alpha}$ is a nonunit in $R$.

Case 2.1. Suppose first that $\operatorname{dim} R=1$ and $R=\mathbb{C}\{z\}$. The proof of this case is the crucial point of the proof of the general case.

Since $\pi$ is nonconstant, using the well-known result in 1-variable complex analysis that a nonconstant holomorphic map is open, we can easily deduce the result. However, we must prove a uniform statement for all 1-dimensional sections of $V$ obtained by intersecting with linear subspaces when $\operatorname{dim} V>1$ and their scheme-theoretic inverse images in $W$. We consider a neighborhood $U$ of $p$ in $V$ defined by points $p^{\prime}$ with $\left|z_{k}\right| \leqslant \delta$ for a suitable $\delta<1$, which we will choose later, and $k$ varies over the set $\{1,2, \ldots, r\}$.

Now, each $a_{i \alpha}$ is a power series in one variable $z$ vanishing at the origin. For any element $f \in J$ let $z^{e}$ be the largest power of $z$ which divides all $a_{\alpha}$, where $f=\sum_{\alpha} a_{\alpha} A^{\alpha}$. Let $J^{\prime}$ be the ideal generated by all $f / z^{e}$ as $f$ varies over elements in $J$. Then $W$ is locally the union of $\{z=0\}$ and the variety $W^{\prime}$ defined by $J^{\prime}$.

For simplicity, assume that $J^{\prime}$ is generated by $f_{1}^{\prime}, \ldots, f_{l}^{\prime}$, and $f_{i}^{\prime}=$ $\Sigma a_{i \alpha}^{\prime} w^{\alpha}$, where $f_{i}^{\prime}=f_{i} / z^{e_{i}}$. No $a_{i 0}^{\prime}$ can be a unit in $R$, for otherwise $J^{\prime}$ is the unit ideal and $W^{\prime}=\phi$. This implies that $\pi$ is constant near $q$. This contradicts the hypothesis that $R \rightarrow S$ is pure (hence injective). However, by construction for each $i$, some $a_{i \alpha}^{\prime}$ with $|\alpha|>0$ is a unit. Arguing as above, we can reduce to the case $m=1$, in which case we get the required result. 
Since we need to prove this result for all 1-dimensional sections of $V$ when $\operatorname{dim} V>1$, we must prove a quantitative statement involving an upper bound on $\left|a_{i j}\right|$.

Again, let $f:=f_{1}$, and let $f=a_{0}+a_{1} w+\cdots$.

Let $\min _{i} \operatorname{ord}_{z}\left(a_{i}\right)=e$. Let $a_{i}^{\prime}:=a_{i} / z^{e}$. Our equation is of the form $a_{0}^{\prime}(z)+a_{1}^{\prime}(z)+\cdots+a_{n}^{\prime}(z) w^{n}+a_{n+1}^{\prime}(z) w^{n+1}+\cdots=0$, such that all the $a_{i}^{\prime}$ are nonunits for $i<n$, and $a_{n}^{\prime}$ is a unit. By the application of Rouchés theorem discussed earlier we deduce the result in this case.

This completes the proof of Case 2.1 when $\operatorname{dim} R=1, m=1$ and $V$ is smooth.

Case 2.2. Now we consider the case when $\operatorname{dim} V=1, m=1$, and $V$ is a singular curve.

The normalization of $\mathcal{O}_{V, p}$ in its quotient field is isomorphic to $\mathbb{C}\{z\}$. Let $V^{\prime} \rightarrow V$ be the normalization. Let $W^{\prime}$ be the fiber product $W \times_{V} V^{\prime}$. Both the morphisms $V^{\prime} \rightarrow V, W^{\prime} \rightarrow W$ are finite, and $W^{\prime} \rightarrow V^{\prime}$ is pure by Lemma 1.4. By taking small open neighborhoods of points in $V^{\prime}$ lying over $p$ we are reduced to the previous case.

Case 3. Now assume that $\operatorname{dim} V \geqslant 2$.

Case 3.1. To explain the main idea we first assume that $V$ is smooth of dimension 2 .

First, we prove two technical results which are useful in the arguments below.

Let $f=a_{0}\left(z_{1}, z_{2}\right)+a_{1}\left(z_{1}, z_{2}\right) w+\cdots+a_{n}\left(z_{1}, z_{2}\right) w^{n}+\cdots$ be a convergent power series in variables $z_{1}, z_{2}, w$, such that $a_{i}\left(z_{1}, z_{2}\right)$ is a nonunit in $R:=\mathbb{C}\left\{z_{1}, z_{2}\right\}$ for every $i$. For any linear homogeneous polynomial $L\left(z_{1}, z_{2}\right)$, consider the ring $R_{0}:=\mathbb{C}\left\{z_{1}, z_{2}\right\} /(L)=\mathbb{C}\{\tau\}$. Clearly, we can take $\overline{z_{1}}$ or $\overline{z_{2}}$ as $\tau$. Assume that $\overline{z_{1}}=\tau$. Let $\overline{a_{i}}$ be the image of $a_{i}$ in $R_{0}$. Then $\overline{a_{i}}$ is a nonunit. Let $e_{i}>0$ be the $\tau$-order of $\overline{a_{i}}$. Consider the set of integers $e_{i}-\left\{\min _{j} e_{j}\right\}$. We assume that for any $L$ we have $e_{0}-\left\{\min _{j} e_{j}\right\}>0$. Let $n_{L}$ be the smallest integer $i$ (depending on $L$ ) such that $e_{i}=\left\{\min _{j} e_{j}\right\}$.

LEMMA 3.5.1. The integers $\min _{j} e_{j}, n_{L}$ are bounded as $L$ varies over the set of all linear homogeneous polynomials, so that at least one $\overline{a_{i}}$ is nonzero in $R_{0}$.

Proof. We consider the ideal $I:=\left(a_{0}, a_{1}, \ldots\right)$. Since $I$ is finitely generated, it follows that $\min _{j} e_{j}$ is bounded as $L$ varies, such that at least one $\overline{a_{i}}$ is nonzero in $R_{0}$. Similarly, $n_{L}$ is bounded above. 
The next result concerns upper bounds on the coefficients of the terms occurring in the application of Rouché's theorem as the linear homogeneous polynomials $L$ vary.

As above, let $g:=\bar{f} / \bar{z}_{1}^{e}=b_{0}+b_{1} w+\cdots$ be a nonunit in $\mathbb{C}\left\{z_{1}, z_{2}, w\right\} /(L)$, with $f \in J$, such that for some $n$ there is a term in $g$ of the form $b_{n} w^{n}$, where $b_{n}$ is a unit in $R$. To start with, by making a suitable unitary (linear) change of coordinates involving $z_{1}, z_{2}$, we can assume that $b_{n}=c_{0}+c_{n}$, where $c_{0}$ is a constant, with $1 / 2 \leqslant\left|c_{0}\right| \leqslant 1$, and $c_{n}$ is a nonunit. In order not to complicate the notation, we assume that $c_{0}=1$. We also assume that $n_{L}$ is the smallest such integer. Then $n_{L}>0$ for all $L$ such that $L$ does not divide $f$. For a fixed $f$, the set of integers $n_{L}$ is bounded above. Hence, for simplicity of exposition, we assume that $n_{L}$ is a constant $n \geqslant 1$.

We can assume that there are suitable $\delta_{1}>0, \delta_{2}>0$ such that $\mid c_{n}+$ $b_{n+1} w+b_{n+2} w^{2}+\cdots \mid<1 / 2$ if $\left|z_{1}\right| \leqslant \delta_{1},\left|z_{2}\right| \leqslant \delta_{1},|w| \leqslant \delta_{2}$.

Since $b_{i}$ is a nonunit for $i<n$, we can assume that $\delta_{1}$ is such that $\left.\left|\left(b_{0}+b_{1} w+\cdots+b_{n-1} w^{n-1}\right)<\right| w\right|^{n} / 2$ when $\left|z_{1}\right| \leqslant \delta_{1},|w|=\delta_{2}$. Now, it follows that $\left|b_{0}+b_{1} w+\cdots\right|<\left|b_{0}+b_{1} w+\cdots+b_{n-1} w^{n-1}+w^{n}\right|$ when $|w|=$ $\delta_{2}$. By Roché's theorem, for any $\left|z_{1}\right| \leqslant \delta_{1},\left|z_{2}\right| \leqslant \delta_{1}$, the numbers of zeros of the equations $b_{0}+b_{1} w+\cdots=0$ and $b_{0}+b_{1} w+\cdots+b_{n-1} w^{n-1}+w^{n}=0$ inside $|w|<\delta_{2}$ are the same. As in the discussion above, this number can be assumed to be $n$.

We must show that the numbers $\delta_{1}, \delta_{2}$ can be chosen independent of $L$.

This can be seen as follows.

Any line $L$ in $\mathbb{C}^{2}$ has the equation $z_{2}=c z_{1}$ for some constant $c$, or $z_{1}=0$. We give the argument assuming that $|c| \leqslant 1$, since if $|c|>1$ then we can take $\overline{z_{2}}=\tau$.

We need to analyze the power series $f\left(z_{1}, c z_{1}, w\right)$ more carefully. If some monomial $\gamma z_{1}^{a} z_{2}^{b}$ occurs in $f$ with $\gamma \in \mathbb{C}$ a nonzero constant, then there is a term $\gamma c^{b} z_{1}^{a+b}$ in $f\left(z_{1}, c z_{1}, w\right)$. The maximum possible number of monomials of total degree $a+b$ in $z_{1}, z_{2}$ is $a+b+1$. For terms $b_{i}$ with $i<n$, if $\left|z_{1}\right| \leqslant \delta_{1}<1 / 2$, then $(a+b+1)\left|c^{b} z_{1}^{a+b}\right| \leqslant\left|(a+b+1) z_{1}^{a+b}\right| \leqslant$ $(a+b+1) \delta^{a+b}$. Since $n$ is bounded, there are at most $n \cdot(a+b+1)$ terms containing $z_{1}^{a+b}$ for $i<n$. Collecting all the terms with the same monomial $z_{1}^{a+b}$, we get $n$ convergent power series of the form $\sum_{l} \gamma_{l} \delta^{l}$, which tend to 0 as $\delta_{1}$ tends to 0 . Hence, $\delta_{1}$ and $\delta_{2}<1$ can be chosen small and independent of $L$. The argument for the series $c_{n}+b_{n+1} w+b_{n+2} w^{2}+\cdots$ is similar but more complicated. In this case we get a convergent power series in variables $\delta_{1}, \delta_{2}$ 
of the form $\sum_{l} \gamma_{l}\left(\delta_{1}\right) \delta_{2}^{l}$, where $\gamma_{l}$ is a convergent power series in $\delta_{1}$. Hence, a similar argument shows that $\left|c_{n}+b_{n+1} w+\cdots\right|<1 / 2$ when $\left|z_{1}\right| \leqslant \delta_{1}, \delta_{2}$ are sufficiently small (independent of $L$ ).

Now, as in the proof when $\operatorname{dim} V=1$, we prove that the image of any Euclidean neighborhood of $O$ in $W$ contains a Euclidean neighborhood of $O$ in $V$.

As before, let $f_{1}, \ldots, f_{l}$ be the equations defining $W$.

Let $J_{i}$ be the ideal generated by all the $a_{i \alpha}$ in $\mathcal{O}_{V, p}$. We consider a prime ideal $\mathfrak{p}$ which defines an irreducible curve $C$ in $V$ which is an irreducible component of a hyperplane section of $V$. It suffices to show that there is an $\epsilon>0$ such that for every such curve $C$ there is an $\epsilon$-neighborhood of $p$ in $C$ which is contained in the image of $\pi$.

For the application of Rouché's theorem, we use $f:=f_{1}$ below, write $f=\sum a_{\alpha} w^{A_{\alpha}}$, and assume that $\left.f\right|_{L}$ is not identically zero for every $L$.

By Lemma 1.3, the extension $R / \mathfrak{p} \subseteq S / \mathfrak{p} S$ is also pure.

Again, first consider the case when $R / \mathfrak{p}$ is smooth. As before, we can assume that $R / \mathfrak{p}=\mathbb{C}\{z\}$.

The functions $\left|a_{i \alpha}\right|_{C}$ have the same upper bound as $\left|a_{i \alpha}\right|$ on $V$. It is possible that for some such $C$ and an $i$ all $a_{i \alpha} \in \mathfrak{p}$. Such an $f_{i}$ can be ignored for the consideration that follows.

We now consider only those $C$ for which $\left.a_{i \alpha}\right|_{C}$ are not all identically zero.

Now, $J_{i} \not \subset \mathfrak{p}$. As above, let $e_{i}$ be the $z$-order of $\overline{f_{i}}$.

Claim. For any $\mathfrak{p}$ such that $J_{i} \not \subset \mathfrak{p}$, the set of integers $e_{i}$ as $L$ varies is finite.

This follows using the primary decomposition of $J_{i}$, and the hypothesis that $\operatorname{dim} V=2$.

Now, $f / z^{e_{i}}=a_{0}^{\prime}+a_{1}^{\prime} w+\cdots+a_{n}^{\prime} w^{n}+\cdots$, where $a_{n}^{\prime}$ is a unit, and no $a_{i}^{\prime}$ is a unit for $i<n$. The set of integers $e_{i}, n_{L}$ is bounded above.

Hence, given any $\eta>0$, we can find a $\delta$ such that whenever $\left|z_{i}\right| \leqslant \delta$, we have $\left|a_{i}^{\prime}\right|<1 / 2$ for $i<n$, and this for all $L$. Similarly, $\mid c_{n}+a_{n+1}^{\prime} w+$ $a_{n+2}^{\prime} w^{2}+\cdots \mid<1 / 2$ if $\left|z_{i}\right| \leqslant \delta,|w| \leqslant \delta$ for all $L$. Therefore, in the application of Rouché's theorem there is a uniform upper bound on the absolute value of at least one $w$ corresponding to any $(z) \in U_{1}$.

Now the proof is similar to the case when $\operatorname{dim} R=1$, and $R$ is smooth.

A similar proof to that above works when $\operatorname{dim} R / \mathfrak{p}=1$, and $R / \mathfrak{p}$ is singular. 
Case 3.2. Now, let $\operatorname{dim} V>2$.

We again consider the ideals $J_{i}$ and irreducible components $C$ of 1 dimensional sections of $V$ with corresponding local $\operatorname{ring} R / \mathfrak{p}$. If some $f_{i} \in \mathfrak{p}$, with the notation above, then such an $f_{i}$ can be ignored for the consideration that follows. If there is a $\mathfrak{p}$ which contains all $J_{i}$, then every point in the curve $C$ is in the image of $\pi$.

Now, assume that not all $f_{i} \in \mathfrak{p}$. The proof is the same as in the twodimensional case. (The proof of the claim is also not difficult.)

We leave the details to the reader.

This completes the proof of Theorem 3.5.

REMARK. In Section 5, we give an example of a graded morphism $\mathbb{C}^{2} \rightarrow \mathbb{C}^{2}$, such that the image of any neighborhood of the origin in the domain contains a neighborhood of the origin in the range (which easily implies the surjectivity of the induced map at germ level), but the morphism is not pure. This shows that the converse of Theorem 3.5 is false.

Theorem 3.5 has an interesting corollary. There exist convergent power series $f_{1}, f_{2}, \ldots, f_{m}$ in variables $z_{1}, \ldots, z_{n}$ with $m>n$ such that there is no convergent power series relation between the $f_{i}$, but there is a formal power series relation between them [4]. This gives an inclusion $R:=\mathbb{C}\left\{f_{1}, f_{2}, \ldots, f_{m}\right\} \subset S:=\mathbb{C}\left\{z_{1}, z_{2}, \ldots, z_{n}\right\}$.

Corollary 3.5.2. Any inclusion $R \subseteq S$ of local analytic domains such that $\operatorname{dim} R>\operatorname{dim} S$ is not pure.

Proof. Suppose that this is a pure extension. By Theorem 3.5, the image of the corresponding analytic map, say $W \rightarrow V$, contains a nonempty open subset $U$ of $V$. By Sard's theorem, the inverse image of a general point of $U$ is a nonempty smooth subvariety of $W$. This is a contradiction, since dim $W<\operatorname{dim} V$.

\section{$\S 4$. Some positive results in special cases}

Coming back to the quasi-finiteness question raised in Section 1, we will see in Section 5 that even a pure morphism of affine planes need not be quasifinite. However, the result is true for subalgebras generated by monomials.

THEOREM 4.1. Let $A$ be a subring of $k^{[n]}\left(:=k\left[x_{1}, \ldots, x_{n}\right]\right)$, generated by the monomials $f_{1}, \ldots, f_{m}$. Suppose that the induced morphism ${ }^{a_{i}}$ : 
$\operatorname{Spec} k^{[n]} \rightarrow \operatorname{Spec} A$ is generically quasi-finite. Then $A \hookrightarrow k^{[n]}$ is pure if and only if $A$ is normal, and the powers $x_{i}^{N}(1 \leqslant i \leqslant n)$ are contained in $A$ for some integer $N>0$. In particular, $k^{[n]}$ is integral over $A$ if $A \hookrightarrow k^{[n]}$ is a pure embedding.

Proof. First, suppose that $A \hookrightarrow k^{[n]}$ is a pure embedding. Then $A$ is also a normal domain. Let $A_{1}, A_{2}, \ldots, A_{m}$ be the $n$-tuples of exponents corresponding to $f_{1}, \ldots, f_{m}$; namely, $A_{i}:=\left(a_{i 1}, a_{i 2}, \ldots, a_{i n}\right)$ when $f_{i}=x_{1}^{a_{i 1}} x_{2}^{a_{i 2}} \cdots x_{n}^{a_{i n}}$. Since ${ }^{a_{i}}$ is generically quasi-finite, the $m \times n$ matrix $A$, whose row vectors are $A_{1}, \ldots, A_{m}$, has rank $n$. After reindexing, if required, we may assume that $A_{1}, \ldots, A_{n}$ are $Q$-independent. Hence, they have maximal rank in the $Q$-vector space generated by $(1,0, \ldots, 0),(0,1,0, \ldots, 0), \ldots,(0,0, \ldots, 1)$. Then, for some integer $r>0$, $r(1,0,0, \ldots, 0)$ lies in the abelian subgroup generated by $A_{1}, A_{2}, \ldots, A_{n}$. After a change of indices, we can write

$$
r(1,0, \ldots, 0)=b_{1} A_{1}+\cdots+b_{s} A_{s}-\left(b_{s+1} A_{s+1}+\cdots+b_{n} A_{n}\right),
$$

where each $b_{i}$ is nonnegative. This means that

$$
f_{1}^{b_{1}} \cdots f_{s}^{b_{s}}=x_{1}^{r} f_{s+1}^{b_{s+1}} \cdots f_{n}^{b_{n}} .
$$

Since $A$ is a pure subring of the integral domain $k^{[n]}$, it follows from Lemma 1.1(2) that $x_{1}^{r} \in A$, and similarly for $x_{2}, \ldots, x_{n}$. Hence, the powers $x_{i}^{N}$ are in $A$ for some $N>0$.

The converse follows from Lemma 1.6.

Generalizing Theorem 4.1, we can then ask whether the following conditions are equivalent.

Question 4.2. Let $A$ be a subring of $k^{[n]}$, generated by the homogeneous polynomials $f_{1}, \ldots, f_{m}$. Suppose that $A$ is normal, and the induced morphism ${ }^{a} i$ :Spec $k^{[n]} \rightarrow$ Spec $A$ is generically quasi-finite. We want to investigate the equivalence of the following three statements.

(1) $A \hookrightarrow k^{[n]}$ is a pure embedding.

(2) The morphism ${ }^{a} i$ is quasi-finite and surjective.

(3) The polynomial ring $k^{[n]}$ is integral over $A$.

We know that any finite morphism is quasi-finite and surjective. Since $A$ is normal, by Lemma 1.6, any surjective and quasi-finite morphism must be pure. Again, it is not difficult to see that any quasi-finite, graded morphism 
is actually finite. Therefore, the question that remains open is whether a polynomial ring is quasi-finite over a graded, pure subring of the same dimension with induced grading.

Let $A \hookrightarrow B$ be an inclusion of affine domains over $k$. In order to approach the question of quasi-finiteness raised in the introduction, we construct the unique subalgebra $C$ of $B$, containing $A$, and satisfying the following properties.

(1) The morphism Spec $C \rightarrow \operatorname{Spec} A$, induced by the inclusion $A \hookrightarrow C$, is a quasi-finite surjective morphism.

(2) $C$ is an affine domain over $k$.

(3) If $C^{\prime} \subset B$ is an affine domain over $k$ such that $A \hookrightarrow C^{\prime}$, and the induced morphism Spec $C^{\prime} \rightarrow \operatorname{Spec} A$ is quasi-finite and surjective, then $C^{\prime} \subseteq C$.

We call $C$ the quasi-finite closure of $A$ in $B$.

THEOREM 4.3. Suppose that $\mathbb{Q}(B)$ is an algebraic extension of $\mathbb{Q}(A)$, and that the morphism $\operatorname{Spec} B \rightarrow \operatorname{Spec} A$ is surjective. Further, assume that $A, B$ are normal. Then the quasi-finite closure $C$ of $A$ in $B$ exists, and satisfies the following properties.

(1) $C$ is an integrally closed domain which is birationally equivalent to $B$.

(2) $C$ is a pure extension of $A$.

Proof. (1) Let $C_{1}$ and $C_{2}$ be finitely generated $k$-subalgebras of $B$ containing $A$, such that Spec $C_{i} \rightarrow$ Spec $A$ is quasi-finite and surjective for $i=1,2$. Let $C_{1} C_{2}$ be the $k$-subalgebra of $B$ generated over $C_{1}$ by $C_{2}$. Any maximal ideal of $C_{1} C_{2}$ is generated by a pair of maximal ideals of $C_{1}, C_{2}$. This implies that $\operatorname{Spec} C_{1} C_{2} \rightarrow \operatorname{Spec} A$ is quasi-finite. It is also surjective since $\operatorname{Spec} B \rightarrow \operatorname{Spec} A$ is surjective.

Now, let $C$ be the $k$-subalgebra of $B$ generated by all finitely generated $k$-subalgebras $C_{i}$ of $B$ containing $A$, such that $\operatorname{Spec} C_{i} \rightarrow \operatorname{Spec} A$ is a quasifinite, surjective morphism.

Since the degree of the field extension $\mathbb{Q}(B) / \mathbb{Q}(A)$ is finite, say $d$, the degree of the extension $\mathbb{Q}(C) / \mathbb{Q}(A) \leqslant d$. Since the normalization of $C_{i}$ in the quotient field of $B$ is finite and surjective over $C_{i}$, we can assume that each $C_{i}$ is normal and birational with $B$. By considering the rings $C_{1}, C_{1} \cdot C_{2}, C_{1}$. $C_{2} \cdot C_{3}, \ldots$ and their normalizations in their quotient field $\mathbb{Q}(B)$, we can assume that $C_{1} \subseteq C_{2} \subseteq C_{3} \subseteq \cdots$ are all quasi-finite extensions. This gives an increasing union of normal birational affine domains $R_{1} \subseteq R_{2} \subseteq \cdots$, which 
are quasi-finite extensions of $A$. It follows that every $R_{i} \subseteq R_{i+1}$ is also quasifinite. By Zariski's main theorem, these inclusions induce open embeddings of corresponding affine schemes. Each $R_{i} \subseteq B$ is birational. Therefore, we can find a suitable hypersurface section $H$ of Spec $B$ defined by $\{h=0\}$ such that $R_{i} \subseteq B[1 / h]$ for each $i$ (this is obvious), and Spec $B \backslash H$ is an affine variety contained in each $\operatorname{Spec} R_{i}$. This implies that the sequence $\operatorname{Spec} R_{i}$ is constant for $i \gg 0$. This proves that $C$ is an affine domain.

Let $b$ be any element of $B$. Since $\mathbb{Q}(B)$ is algebraic over $\mathbb{Q}(A)$, there exists a nonzero element $a \in A$ such that $a b$ is integral over $A$. Then $A[a b]$, which is a $k$-subalgebra generated by $a b$ over $A$, is contained in $C$ because $\operatorname{Spec} A[a b] \rightarrow \operatorname{Spec} A$ is a finite morphism. Hence, $A[a b] \subseteq C$. This implies that $b \in \mathbb{Q}(C)$, and hence $\mathbb{Q}(C)=\mathbb{Q}(B)$. If $b$ is an integral element of $B$ over $C$, then $C[b]$ is a $k$-subalgebra of $B$, such that $\operatorname{Spec} C[b] \rightarrow \operatorname{Spec} C$ is a finite morphism. Hence, Spec $C[b] \rightarrow \operatorname{Spec} A$ is a quasi-finite surjective morphism. Hence, $C[b]=C$, implying that $b \in C$.

(2) This follows from the quasi-finiteness of $\operatorname{Spec} C \rightarrow \operatorname{Spec} A$.

With the notation as in Theorem 4.3 , set $Y:=\operatorname{Spec}(B), X:=\operatorname{Spec}(A)$, and $f: Y \rightarrow X$ is the morphism induced by the inclusion $A \hookrightarrow B$. We assume that $A$ and $B$ are normal. Let $F$ be the closed set of $X$ consisting of the points $x$, such that $f^{-1}(x)$ contains an irreducible component of positive dimension. Let $\Delta$ be the union of all irreducible components of codimension one in $f^{-1}(F)$, and let $Y^{\prime}:=Y \backslash \Delta$. Then $Y^{\prime}$ is an affine scheme Spec $B^{\prime}$, where $B^{\prime}$ is the ideal quotient $(B: J):=\left\{f \in \mathbb{Q}(B) \mid f J^{n} \subseteq B\right.$ for some $\left.n\right\}$, where $J$ is the defining ideal of $\Delta$. In fact, since $B$ is normal, $B^{\prime}$ is the intersection of $B_{\mathfrak{q}}$, where $\mathfrak{q}$ ranges over all prime ideals of height one of $B$ such that $V(\mathfrak{q}) \nsubseteq \Delta$. We prove the following result.

THEOREM 4.4. Let the notations and assumptions be the same as in Theorem 4.3 and as above. Suppose that $C$ is finitely generated over $k$. Then $Y^{\prime}$ is an open set of $\operatorname{Spec} C$, and the restriction $\left.f\right|_{Y^{\prime}}: Y^{\prime} \rightarrow X$ is almost surjective, that is, the image $f\left(Y^{\prime}\right)$ contains all codimension one points of $X$.

Proof. Let $\mathfrak{p}$ be a prime ideal of height 1 of $A$. Since $f: Y \rightarrow X$ is surjective, there exists a prime ideal $\mathfrak{q}$ of $B$ such that $\mathfrak{q} \cap A=\mathfrak{p}$. Then $\mathfrak{q}$ has height 1 . In fact, let $\mathfrak{P}=\mathfrak{q} \cap C$. Since $\operatorname{Spec}(C) \rightarrow \operatorname{Spec}(A)$ is a quasifinite morphism, $\mathfrak{P}$ has height 1 . Note that $B$ and $C$ are birational, and $B_{\mathfrak{q}}$ dominates $C_{\mathfrak{P}}$. Since $C_{\mathfrak{P}}$ is a discrete valuation ring, we have $B_{\mathfrak{q}}=C_{\mathfrak{P}}$. Note that, if $\mathfrak{p}$ ranges over all prime ideals of height 1 in $A$, then $\mathfrak{q}$, lying 
over $\mathfrak{p}$, will range over all prime ideals of height 1 in $B$ such that $V(\mathfrak{q}) \nsubseteq \Delta$. Therefore, the open set $Y^{\prime}$ is contained in Spec $(C)$. The above argument shows that $\left.f\right|_{Y^{\prime}}$ is almost surjective.

Let $S$ be the polynomial ring in $n$-variables over $k$, and let $G$ be a reductive algebraic subgroup of $G L(n, k)$, acting naturally on $S$. Then the ring of invariants $R:=S^{G}$ is a graded subring of $S$. Let $\mathfrak{m}, \mathfrak{n}$ be the irrelevant maximal ideals of $R, S$ respectively.

The next result may be well known to the experts.

Proposition 4.5. $R_{\mathfrak{m}}$ is a pure subring of $S_{\mathfrak{n}}$.

Proof. The Reynold's operator sends $f \in S$ to $f_{0} \in R$ by a suitable integration over a maximal compact subgroup $K$ of $G$. An element $f \in S$ vanishes at the origin if and only if $f_{0}$ vanishes at the vertex in $\operatorname{Spec} R$. Any element in $S_{\mathfrak{n}}$ is of the form $f / g$, where $f, g$ have no common factor, and $g$ does not vanish at the origin. Since $S$ has no nonconstant units, such a representation of an element of $S_{\mathfrak{n}}$ is unique up to a nonzero constant. Therefore, the Reynold's operator can be uniquely extended to an $R_{\mathfrak{m}^{-}}$ module homomorphism of $S_{\mathfrak{n}}$ to $R_{\mathfrak{m}}$ by sending $f / g$ to $f_{0} / g_{0}$. This shows that $R_{\mathfrak{m}}$ is a direct summand of $S_{\mathfrak{n}}$, and hence a pure subring.

\section{§5. Examples, and more observations on pure extensions}

To start with, let us consider the following two morphisms of affine domains, which manifest the subtlety of pure extensions.

EXAMPLE 5.1.

$$
\phi: k[x, y] \hookrightarrow \frac{k[x, y, z]}{\left(x z^{2}-y z-x y\right)}
$$

and

$$
\psi: k[x, y] \hookrightarrow \frac{k[x, y, z]}{\left(x z^{2}-y z-x y^{2}\right)} .
$$

Then $\phi$ is not pure, but $\psi$ is pure.

Proof. To show that $\phi$ is not pure, look at the ideal $I \subseteq k[x, y]$, generated by $x^{4}$ and $y^{4}+3 x^{2} y^{3}+x^{4} y^{2}$. Then $x y^{4}+2 x^{3} y^{3} \notin I$, whereas $x y^{4}+2 x^{3} y^{3} \in$ $I\left(k[x, y, z] /\left(x z^{2}+y z+x y\right)\right) \cap k[x, y]$ because of the relation

$$
x^{4} z^{5} \in\left(y^{4}+3 x^{2} y^{3}+x^{4} y^{2}\right) z+\left(x y^{4}+2 x^{3} y^{3}\right) .
$$


The relation is obtained by successive substitution $y z+x y$ for $x z^{2}$. The purity of $\psi$, on the other hand, is a consequence of the following lemma.

LEMma 5.2. Let $f$ be a polynomial in $k[x, y]$, divisible by $y^{2}$. Then the map

$$
\Psi: k[x, y] \longrightarrow \frac{k[x, y, z]}{\left(x z^{2}+y z+f\right)}
$$

is pure.

Proof. First, note that the associated morphism of schemes is surjective, since it is surjective on the closed points. Therefore, by local criteria for purity as in Lemma 1.3, it is sufficient to check that the induced local morphism over the origin

$$
\Psi_{0}: k[x, y]_{(x, y)} \longrightarrow \frac{k[x, y, z]}{\left(x z^{2}+y z+f\right)} \otimes k[x, y]_{(x, y)}
$$

is pure, as, over any point other than the origin, the morphism is quasifinite, and hence is pure by Lemma 1.6. Again, in view of the observation that any partially pure point is pure, it suffices if we can show that the induced map

$$
\Psi_{0}^{\prime}: k[x, y]_{(x, y)} \longrightarrow\left(\frac{k[x, y, z]}{\left(x z^{2}+y z+f\right)}\right)_{(x, y, z)}
$$

is pure. However, the polynomial $x z^{2}+y z+f$, as a polynomial in $z$, has roots in $k[[x, y]]$ since $y^{2} \mid f$. Therefore, the corresponding map of completions

$$
\hat{\Psi}_{0}^{\prime}: k[[x, y]] \longrightarrow \frac{k[[x, y, z]]}{\left(x z^{2}+y z+f\right)}
$$

is pure. Consequently, in view of the commutative diagram,

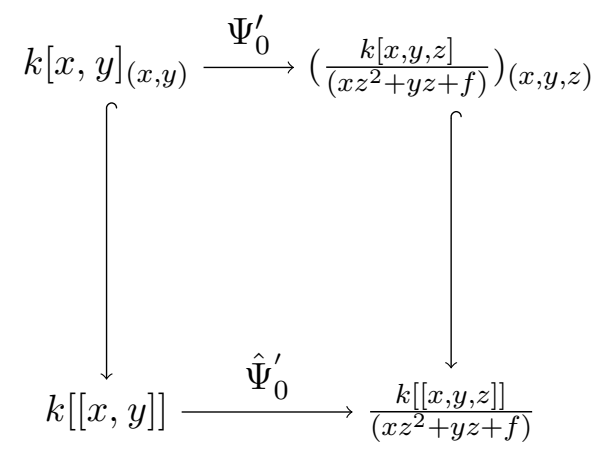


Lemma 2.4 implies that $\Psi_{0}^{\prime}$ is pure. Hence, $\Psi$ is pure. This completes the proof of Lemma 5.2 and shows that $\psi$ is a pure morphism.

The notion of pure morphism generalizes the ring of invariants of a polynomial ring under some group actions. If a finite group $G$ acts on $A:=k\left[x_{1}, x_{2}, \ldots, x_{n}\right]$, then $A$ is finite over the ring of invariants $A^{G}$. However, in the case of a pure extension of normal, affine domains of same dimension, the map may not even be quasi-finite.

EXAMPLE 5.3. (A pure morphism of affine surfaces need not be quasifinite) Let us consider the affine morphism

$$
\mathbb{A}^{2} \stackrel{f}{\rightarrow} \mathbb{A}^{2}, \quad \text { given by }(x, y) \mapsto(x(x+1), x y) \text {. }
$$

It is easy to see that $f$ is surjective. Moreover, it is quasi-finite outside the origin, and the fiber over the origin is the union of the line $L:=$ $\left\{(x, y) \in \mathbb{A}^{2} \mid x=0\right\}$ and the point $(-1,0)$. Therefore, removing $L$, we get a morphism, say $\widetilde{f}$, between normal affine varieties, which is quasi-finite and surjective.

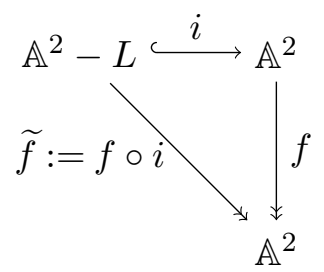

By Lemma 1.6, the morphism $\tilde{f}$ is a pure morphism of affine normal varieties. Hence, $f$ is a pure morphism by Lemma 1.1(1). However, $f$ is not quasi-finite.

REMARK. In the following commutative diagram:

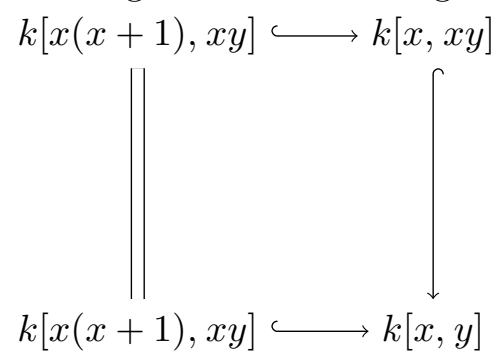

$k[x(x+1), x y] \hookrightarrow k[x, y]$ is pure, and hence, by Lemma $1.1, k[x(x+1), x y]$ $\hookrightarrow k[x, x y]$ is also pure. However, since $k[x, x y]$ is birational to $k[x, y]$, 
Corollary 2.3.1 implies that $k[x, x y] \hookrightarrow k[x, y]$ cannot be pure. This should be contrasted with Lemma 2.5.

We saw in Example 5.3 that the local morphism at the origin is pure in spite of the fiber over it containing a line. However, in this case, the fiber also contains an isolated point, i.e., a component of "correct" dimension. Therefore, it is natural to ask whether the purity is due to the presence of the "correct" dimensional component in the fiber. Therefore, we pose the following question.

Question. Let $\phi: X \longrightarrow Y$ be a morphism of normal affine varieties. Suppose that $y \in Y$ is pure. Is it true that $\phi^{-1}(y)$ must contain a component of "correct" dimension, i.e., of dimension equal to $\operatorname{dim} X-\operatorname{dim} Y$ ?

Unfortunately, this question has a negative answer, as the following example shows.

ExAMPLE 5.4. Let the multiplicative group $\mathbb{C}^{*}$ act on the polynomial $\operatorname{ring} S:=\mathbb{C}[X, Y, Z, W]$ by $\rho_{\lambda}(X, Y, Z, W)=\left(\lambda X, \lambda Y, \lambda^{-1} Z, \lambda^{-1} W\right)$. By the use of Reynold's operator, it is well known that the ring of invariants $R:=\mathbb{C}[X Z, X W, Y Z, Y W]$ is a direct summand of $S$, and hence $R \subset S$ is a pure extension. The inverse image of the vertex of $\operatorname{Spec} R$ in $\operatorname{Spec} S$ is the union of two linear subvarieties $\{X=Y=0\}$ and $\{Z=W=0\}$, each having dimension 2 , which is larger than $\operatorname{dim} S-\operatorname{dim} R=1$.

ExAMPLE 5.5. (The pure locus is not open in general) Consider the affine morphism

$$
\theta: \mathbb{A}^{3} \longrightarrow \mathbb{A}^{2}, \quad \text { given by }(x, y, z) \mapsto(x z, y z) .
$$

The fiber over $(0,0) \in \mathbb{A}^{2}$ is the union of the line $\left\{(x, y, z) \in \mathbb{A}^{3} \mid x=y=0\right\}$ and the plane $\left\{(x, y, z) \in \mathbb{A}^{3} \mid z=0\right\}$. Now $\mathcal{O}_{\mathbb{A}^{2},(0,0)} \longrightarrow \mathcal{O}_{\mathbb{A}^{3},(0,0,0)}$ is pure by Proposition 4.5 , since $k[x z, y z]$ is the ring of invariants of $k[x, y, z]$ under the action of $k^{*}$, given by $(\lambda,(x, y, z)) \mapsto\left(\lambda x, \lambda y, \lambda^{-1} z\right)$. However, it follows from the following Theorem 5.6 that there exist points in $\mathbb{A}^{3}$, arbitrarily close to the origin, such that $\operatorname{Spec} \mathcal{O}_{\mathbb{A}^{3},(x, y, z)} \longrightarrow \operatorname{Spec} \mathcal{O}_{\mathbb{A}^{2}, \theta(x, y, z)}$ is not surjective, and consequently $\operatorname{Spec} \mathcal{O}_{\mathbb{A}^{3},(x, y, z)} \longrightarrow \operatorname{Spec} \mathcal{O}_{\mathbb{A}^{2}, \theta(x, y, z)}$ is not pure.

TheOREm 5.6. Let $f: X \longrightarrow Y$ be a morphism of affine varieties, with $y \in Y$, such that $\operatorname{dim} f^{-1}(y)>\operatorname{dim} X-\operatorname{dim} Y$. Then, for any irreducible component of highest dimension, say $Z$, of $f^{-1}(y)$, there exists a nonempty Zariski-open set $U \subseteq Z$, such that for all $x \in U$, the induced morphism $\operatorname{Spec} \mathcal{O}_{X, x} \longrightarrow \operatorname{Spec} \mathcal{O}_{Y, y}$ is not surjective. 
Proof. The proof is left to the reader as an exercise.

Theorem 5.6 has the following two interesting corollaries.

COROLlary 5.6.1. Any strongly pure morphism of affine algebraic varieties is equidimensional.

COROllary 5.6.2. The nonequidimensional pure extension

$$
k[x z, y z] \hookrightarrow k[x, y, z]
$$

shows that a pure morphism need not be strongly pure.

Note. If we choose the underlying field to be the field of complex numbers $\mathbb{C}$, then the above proof also tells us that the pure locus is not even open in Euclidean topology, as any nonempty Zariski-open set in a variety is dense in the Euclidean topology.

5.7. The set of completely pure points may not be open. Let $Y:=\mathbb{A}^{3}$, $X:=Y \times F$, where $F \cong \mathbb{A}^{3}$. Let $L \in Y$ be a line, and let $L^{\prime}:=L \times\{0\} \subset X$, which maps isomorphically onto $L$. Let $f: \tilde{X} \rightarrow X$ be the blow-up with center $L^{\prime}$, and let $Z:=f^{-1}\left(L^{\prime}\right)$. Then, $Z \cong L^{\prime} \times \mathbb{P}^{4}$. Every fiber of the composite morphism $\tilde{X} \rightarrow Y$ for a point $y$ in $L$ is a union of the proper transform of $F_{y}$ and a variety $V_{y}$ isomorphic to $\mathbb{P}^{4}$, where $F_{y}$ is the fiber over $y$ of the morphism $X \rightarrow Y$. Let $X_{0}:=\tilde{X} \backslash V_{O}$, where $O \in Y$ is the origin. Now, the natural morphism $X_{0} \rightarrow Y$ has the property that the fiber over $O$ has dimension 3, and for any $y \in Y$ with $y \neq O$, the fiber over $y$ contains an irreducible component of dimension 4. The point $O \in Y$ is completely pure for the morphism $X_{0} \rightarrow Y$, but other points in $L$ are not pure for this morphism. This shows that the set of completely pure points may not be open.

REMARK. The above example also shows that in Grothendieck's theorem on generic flatness, for a morphism $X \rightarrow Y$, the set of points $y$ in $Y$ such that the morphism is flat over $y$ may not be open. It also shows that the dimension of a fiber may not be an upper-semicontinuous function on $Y$.

In Section 1, we saw that pure extensions are preserved under tensor products. However, as the following example shows, this fails to hold true for the composite of subalgebras.

EXAMPLE 5.8. Let $k[x, y] \subseteq k[x, y, z, w] /\left(x^{2} z+y w+x\right)$. Now consider the two $k[x, y]$-subalgebras $B_{1}:=k[x, y, z]$ and $B_{2}:=k[x, y, w]$. Then, each 
$B_{i}$ is a pure extension of $k[x, y]$. However, $k[x, y, z, w] /\left(x^{2} z+y w+x\right)=$ $B_{1} B_{2}$ is not a pure extension of $k[x, y]$.

Proof. If $I$ is defined to be the ideal generated by $x^{2}$ and $y$ in $k[x, y]$, then

$$
x \in I\left(\frac{k[x, y, z, w]}{\left(x^{2} z+y w+x\right)}\right) \cap k[x, y] \backslash I .
$$

REMARK. If $A \subseteq B$ are commutative rings, then a simple application of Zorn's lemma shows that there exist maximal (cyclically) pure extensions of $A$ in $B$. However, the above example shows that there may be several such maximal extensions, with none being 'the maximum' one. Consequently, any attempt to define notions like (cyclically) pure closure proves futile.

Lemma 1.9 tells us that, if $A$ is a Noetherian complete local ring, then a ring extension $A \subseteq B$ is pure if and only if $A$ is a direct summand of $B$. The next example shows that a pure extension of Noetherian rings, in general, need not be a direct summand.

Lemma 5.9. Let $A$ be an integral domain, and let $A[x, y]$ be the polynomial ring in two variables over $A$. Then,

$$
A[x] \hookrightarrow \frac{A[x, y]}{\left(x y^{2}-y+1\right)}
$$

is faithfully flat by Nagata's criteria of faithful flatness [14], and hence pure by Lemma 1.7. However, this extension is not a direct summand.

Proof. If possible, let

$$
\theta: \frac{A[x, y]}{\left(x y^{2}-y+1\right)} \longrightarrow A[x]
$$

be an $A[x]$-linear map, and denote the image of $\theta\left(Y^{n}\right)$ by $\theta_{n}$. Then, for any $n \geqslant 0$, we have

$$
x \theta_{n+2}=\theta_{n+1}-\theta_{n} .
$$

First, note that $\theta_{1}$ must have a positive degree, as otherwise either $\theta_{2}$ or $\theta_{3}$ is not solvable. Again, $\operatorname{deg} \theta_{2}=\operatorname{deg} \theta_{3}=\operatorname{deg} \theta_{1}-1$. A routine inductive argument shows that, for any $n \geqslant 1$,

$$
\operatorname{deg} \theta_{2 n} \leqslant \operatorname{deg} \theta_{1}-n, \text { as well as } \operatorname{deg} \theta_{2 n+1} \leqslant \operatorname{deg} \theta_{1}-n .
$$


Since the degree of any nonzero polynomial is always nonnegative, the $\theta_{n}$ will eventually be 0 for all $n \gg 0$. Let $n_{0}$ be a positive integer such that $\theta_{n_{0}-1} \neq 0$ and $\theta_{n}=0$ for all $n \geqslant n_{0}$. Then,

$$
x \theta_{n_{0}+1}=\theta_{n_{0}}-\theta_{n_{0}-1} .
$$

This is a contradiction.

The following example shows that we can even get a pure embedding of algebraic local domains which is not a direct summand.

EXAMPLE 5.10. Let $k$ be an algebraically closed field of characteristic 0. Let us consider the following example:

$$
k[x]_{(x)} \hookrightarrow\left(\frac{k[x, y]}{\left(x y^{2}-y+x\right)}\right)_{(x, y)} .
$$

The extension is faithfully flat by Nagata's criteria of faithful flatness [14], and hence pure by Lemma 1.7. However, as we will see, it is not a direct summand.

Proof. The polynomial $x y^{2}-y+x$, as a polynomial in $y$ over $k[x]$, splits in $k[[x]]$, with the roots being

$$
y=\frac{-1 \pm \sqrt{1-4 x^{2}}}{2 x} .
$$

Therefore, taking the '-' sign, we get a root, say $x y_{0}$, with $y_{0}:=1+x^{2}+$ ... . If possible, let

$$
\theta:\left(\frac{k[x, y]}{\left(x y^{2}-y+x\right)}\right)_{(x, y)} \rightarrow k[x]_{(x)}
$$

be a $k[x]_{(x)}$-linear map. A moment's reflection will convince the reader that $\left(\frac{k[x, y]}{\left(x y^{2}-y+x\right)}\right)_{(x, y)}$ is a DVR, and

$$
i:\left(\frac{k[x, y]}{\left(x y^{2}-y+x\right)}\right)_{(x, y)} \longrightarrow k[[x]], \quad \text { with } y \mapsto x y_{0},
$$

is an embedding of local rings. Let $B_{\mathfrak{n}}$ be the image of $\left(\frac{k[x, y]}{\left(x y^{2}-y+x\right)}\right)_{(x, y)}$ under $i$. Then,

$$
\theta \circ i^{-1}: B_{\mathfrak{n}} \rightarrow k[x]_{(x)}
$$


is also $k[x]_{(x)}$-linear. Note that the $\mathfrak{n} B_{\mathfrak{n}}$-adic topology of $B_{\mathfrak{n}}$ coincides with the topology induced by the $x k[[x]]$-adic topology of $k[[x]]$. Since $k[x]_{(x)}$ is dense in $k[[x]]$ with respect to the $x k[[x]]$-adic topology, it follows that $k[x]_{(x)}$ is also dense in $B_{\mathfrak{n}}$ with respect to the $\mathfrak{n} B_{\mathfrak{n}}$-adic topology. Moreover, any $k[x]_{(x)}$-linear endomorphism of $B_{\mathfrak{n}}$ is continuous with respect to the $\mathfrak{n} B_{\mathfrak{n}^{-}}$ adic topology. So the $k[x]_{(x)}$-linear map $\theta \circ i^{-1}$, being identity on a dense subset $k[x]_{(x)}$, must be identity on $B_{\mathfrak{n}}$. This is a contradiction since $x y_{0} \in B_{\mathfrak{n}}$ is not contained in $k[x]_{(x)}$.

In the final two examples, we deal with complex affine varieties. Suppose that $X$ and $Y$ are affine cones, i.e., affine varieties given by homogeneous prime ideals, of the same dimension, and $f: X \longrightarrow Y$ is a surjective morphism given by homogeneous polynomials. In this set-up, the question of purity over the origin plays a key role in determining the purity of $f$. Therefore, it is natural to ask how the following three properties are related.

(1) The induced local morphism at the origin $f_{0}^{*}: \mathcal{O}_{Y, 0} \longrightarrow \mathcal{O}_{X, 0}$ is pure.

(2) Given any Euclidean open ball around the origin in $X, f(X)$ contains a Euclidean open ball around the origin in $Y$.

(3) The associated morphism of stalks at the origin $\widetilde{f}_{0}: \operatorname{Spec} \mathcal{O}_{X, 0} \longrightarrow$ Spec $\mathcal{O}_{Y, 0}$ is surjective.

By Lemma 1.1, purity implies local surjectivity of the corresponding morphism of schemes. By Theorem 3.5, (1) implies (2).

Since any nonempty Euclidean open set in a variety is dense in its Zariskitopology, it is easy to see that (2) implies (3). The last property, as one might expect, is indeed weaker than the other two. In the next example, we see that (3) does not imply either (1) or (2). Finally, the last example shows that, even if the image of any Euclidean ball at the origin contains an open set around the origin, the induced local morphism may not be pure.

EXAMPLE 5.11. Let us consider a morphism of affine planes

$$
\theta: \mathbb{A}^{2} \longrightarrow \mathbb{A}^{2}, \quad \text { given by }(x, y) \mapsto\left(x y^{2}, x^{2}(x+y)\right)
$$

Clearly, $\theta$ is surjective. Considering the factorizations of polynomials in the variables $x y^{2}$ and $x^{2}(x+y)$ in $k[x, y]$, it is also not difficult to see that even

$$
\text { Spec } k[x, y]_{(x, y)} \longrightarrow \text { Spec } k\left[x y^{2}, x^{2}(x+y)\right]_{\left(x y^{2}, x^{2}(x+y)\right)}
$$


is surjective, which, since the rings under consideration are two-dimensional, also implies that the going-down property holds for

$$
k\left[x y^{2}, x^{2}(x+y)\right]_{\left(x y^{2}, x^{2}(x+y)\right)} \hookrightarrow k[x, y]_{(x, y)} .
$$

After a suitable change of variables, one can show that $k\left[x y^{2}, x^{2}(x+y)\right] \subseteq$ $k\left[x y^{2}, x^{2}(x+y), y^{3}\right]$ is not pure, implying that $k\left[x y^{2}, x^{2}(x+y)\right] \subseteq k[x, y]$ is also not pure. Finally, to make sense of the second property, we confine ourselves to complex affine varieties. In order to show that $\theta$ does not satisfy (2), first note that since the map is homogeneous, it is sufficient to prove that there exists an open ball, say $\mathbb{B}$, around the origin such that $\theta(\mathbb{B})$ does not contain any open set around the origin.

Therefore, let us define $U:=\mathbb{B}((0,0), 1 / 2)$. If possible, let $V$ be an open set around the origin contained in $f(U)$. Choose any $(a, a) \in V \backslash\{(0,0)\}$. Then, a routine computation shows that for any sufficiently large $n$, the points of the form $(a / n, a)$ are not in the image of $U$. Hence, $f(U)$ does not contain any open set around the origin.

The last example shows that (3) is actually weaker than (1).

Example 5.12. Let $R:=\mathbb{C}\left[X\left(Y^{2}-X^{2}\right), X^{2} Y\right] \subseteq S:=\mathbb{C}[X, Y]$. This gives a morphism $\pi: \mathbb{C}^{2} \rightarrow \mathbb{C}^{2}$. Since the line $\{X=0\}$ maps to the origin, $\pi$ is not quasi-finite. It is not difficult to show that the image of any neighborhood of the origin contains a neighborhood of the origin. However, the morphism is not pure.

\section{$\S 6$. Open problems}

In this section we gather together several questions about pure extensions for which the authors do not know answers.

Question 1. Let $A$ be an $n$-dimensional affine graded $k$-subalgebra of the polynomial ring $k\left[X_{1}, X_{2}, \ldots, X_{n}\right]$. If this is a pure extension, then is the corresponding morphism of schemes quasi-finite?

Question 2. Let $A \subset k[X, Y]$ be a 2-dimensional $k$-subalgebra of the polynomial ring $k[X, Y]$ such that $A$ is a direct summand of $k[X, Y]$ as an $A$-module. Is the corresponding morphism of schemes quasi-finite?

Question 3. Let $A \subset B$ be affine domains. Suppose that for some maximal ideal $\mathfrak{m} \subset A$ the extension $A_{\mathfrak{m}} \subset B_{\mathfrak{m}}$ is pure. Is there a maximal ideal $\mathfrak{n} \subset B$ lying over $\mathfrak{m}$ such that $A_{\mathfrak{m}} \subset B_{\mathfrak{n}}$ is pure? 
Question 4. Let $A \subset B$ be a pure extension of affine $k$-domains (or algebraic local domains) such that this is a pure extension. Assume that $A$ is normal. Is the extension $A \subset \bar{B}$ also pure, where $\bar{B}$ is the integral closure of $B$ in its quotient field?

Question 5. Let $A \subset B$ be affine $k$-domains. Consider the set of prime ideals $\mathfrak{p} \subset A$ such that $A_{\mathfrak{p}} \subset B_{\mathfrak{p}}$ is a pure extension. Is this set open in $\operatorname{Spec} A$ ?

Question 6. Let $A \subset B$, as in Question 5 above. Consider the set of prime ideals $\mathfrak{p} \subset A$ such that for some prime ideal $\mathfrak{q} \subset B$ lying over $\mathfrak{p}$ the extension $A_{\mathfrak{p}} \subset B_{\mathfrak{q}}$ is pure. Is this set open in Spec $A$ ?

\section{REFERENCES}

[1] S. S. Abhyankar, Resolution of Singularities of Embedded Algebraic Surfaces, Academic Press, 1966.

[2] M. Artin, Algebraic approximation of structures over complete local rings, Publ. Math. Inst. Hautes Études Sci. 36 (1969), 23-58.

[3] P. Bonnet, Surjectivity of quotient maps for algebraic $(\mathbb{C},+)$-actions and polynomial maps with contractible fibers, Transform. Groups 7 (2002), 3-14.

[4] A. M. Gabrièlov, The formal relations among analytic functions, Funct. Anal. Appl. 5 (1971), 318-319.

[5] M. Hashimoto, A pure subalgebra of a finitely generated algebra is finitely generated, Proc. Amer. Math. Soc. 133(8) (2005), 2233-2235.

[6] M. Hochster, Cyclic purity versus purity in excellent Notherian rings, Trans. Amer. Math. Soc. 231 (1977), 463-488.

[7] M. Hochster and J. L. Roberts, Rings of invariants of reductive groups acting on regular rings are Cohen-Macaulay, Adv. Math. 13 (1974), 115-175.

[8] M. Hochster and J. L. Roberts, The purity of the Frobenius and local cohomology, Adv. Math. 21 (1976), 117-172.

[9] M. Hochster, Contracted ideals from integral extensions of regular rings, Nagoya Math. J. 51 (1973), 25-43.

[10] S. Kaliman, Free $\mathbb{C}_{+}$-actions on $\mathbb{C}^{3}$ are translations, Invent. Math. 156 (2004), 163-173.

[11] H. Matsumura, Commutative Algebra, 2nd ed., Mathematics Lecture Note Series 56 Benjamin-Cummings Pub Co., 1980.

[12] M. Miyanishi, Normal affine subalgebras of a polynomial ring, Algebraic and Topological Theories - to the memory of Dr. Takehiko MIYATA, Kinokuniya, 1985, $37-51$.

[13] M. Nagata, Local Rings, Interscience Tracts in Pure and Applied Mathematics, John Wiley and Sons, 1962.

[14] M. Nagata, Flatness of an extension of a commutative ring, J. Math. Kyoto Univ. 9 (1969), 439-448.

[15] R. B. Warfield Jr., Purity and algebraic compactness for modules, Pacific J. Math. 28 (1969), 699-719. 
S. Chakraborty

School of Mathematics

Tata Institute of Fundamental Research

Homi Bhabha Road

Mumbai 400005

India

sagnik@math.tifr.res.in

R. V. Gurjar

School of Mathematics

Tata Institute of Fundamental Research

Homi Bhabha Road

Mumbai 400005

India

gurjar@math.tifr.res.in

M. Miyanishi

Research Center for Mathematical Sciences

Kwansei Gakuin University

2-1 Gakuen

Sanda

Hyogo 669-1337

Japan

miyanisi@amber.plala.or.jp 